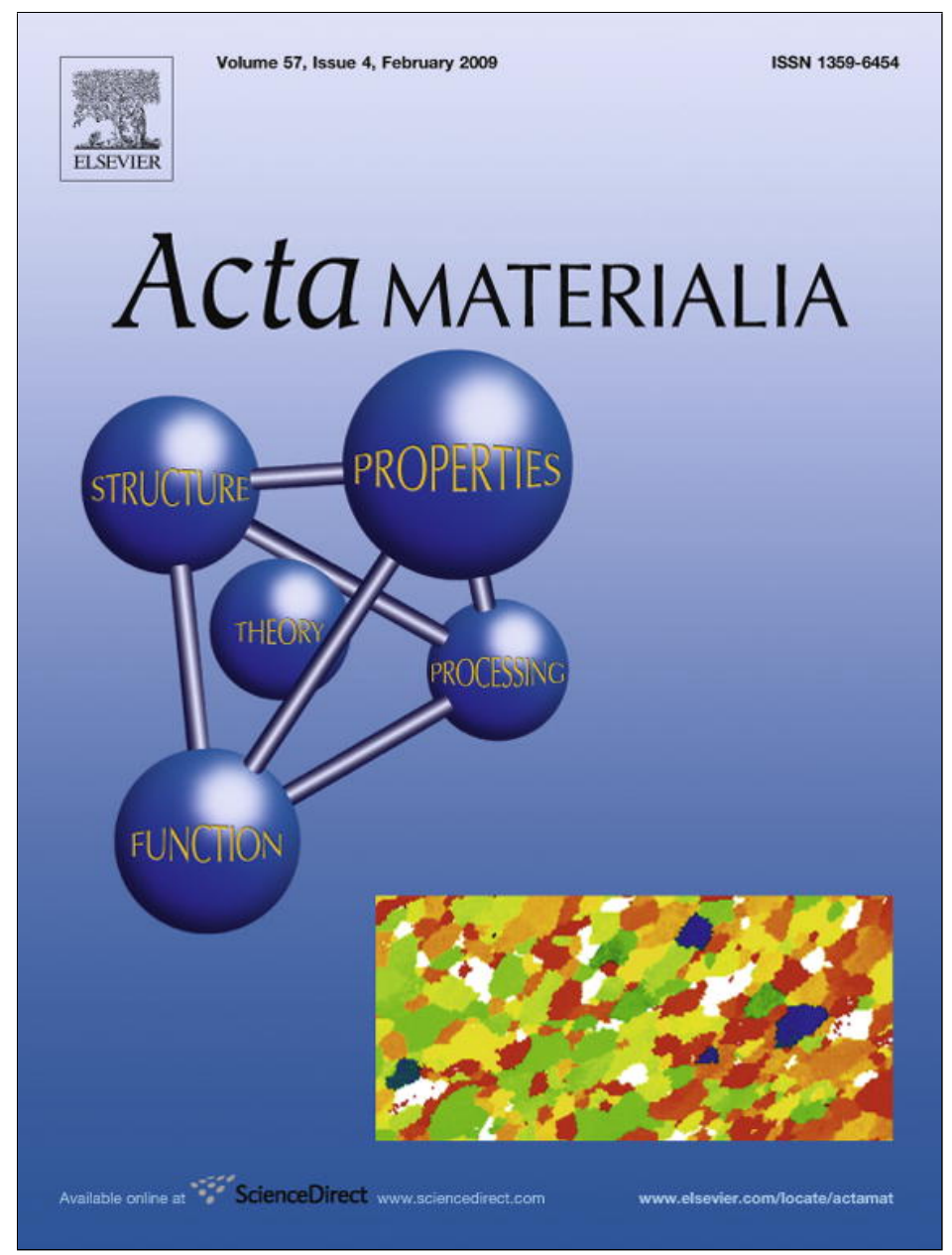

This article appeared in a journal published by Elsevier. The attached copy is furnished to the author for internal non-commercial research and education use, including for instruction at the authors institution and sharing with colleagues.

Other uses, including reproduction and distribution, or selling or licensing copies, or posting to personal, institutional or third party websites are prohibited.

In most cases authors are permitted to post their version of the article (e.g. in Word or Tex form) to their personal website or institutional repository. Authors requiring further information regarding Elsevier's archiving and manuscript policies are encouraged to visit:

http://www.elsevier.com/copyright 


\title{
Solidification microstructures and solid-state parallels: Recent developments, future directions
}

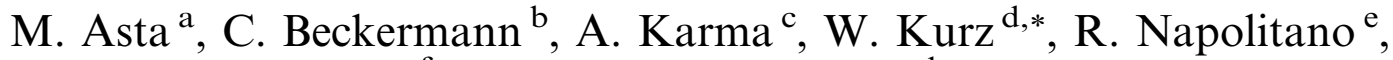 \\ M. Plapp ${ }^{f}$, G. Purdy ${ }^{\mathrm{g}}$, M. Rappaz ${ }^{\mathrm{d}}$, R. Trivedi ${ }^{\mathrm{e}}$ \\ ${ }^{a}$ Department of Chemical Engineering and Materials Science, University of California at Davis, Davis, CA 95616, USA \\ ${ }^{\mathrm{b}}$ Department of Mechanical and Industrial Engineering, The University of Iowa, Iowa City, IA 52242, USA \\ ${ }^{\mathrm{c}}$ Department of Physics and Center for Interdisciplinary Research on Complex Systems, Northeastern University, Boston, MA 02115, USA \\ ${ }^{\mathrm{d}}$ Institute of Materials, Ecole Polytechnique Fédérale de Lausanne (EPFL), 1015 Lausanne, Switzerland \\ ${ }^{\mathrm{e}}$ Department of Materials Science and Engineering, Iowa State University, and Ames Laboratory USDOE, Ames, IA 50011, USA \\ ${ }^{\mathrm{f}}$ Physique de la Matière Condensée, Ecole Polytechnique, CNRS, 91128 Palaiseau, France \\ ${ }^{\mathrm{g}}$ Department of Materials Science and Engineering, McMaster University, Hamilton, Ont., Canada L8S $4 L 7$
}

Received 11 January 2008; received in revised form 2 October 2008; accepted 2 October 2008

Available online 26 December 2008

\begin{abstract}
Rapid advances in atomistic and phase-field modeling techniques as well as new experiments have led to major progress in solidification science during the first years of this century. Here we review the most important findings in this technologically important area that impact our quantitative understanding of: (i) key anisotropic properties of the solid-liquid interface that govern solidification pattern evolution, including the solid-liquid interface free energy and the kinetic coefficient; (ii) dendritic solidification at small and large growth rates, with particular emphasis on orientation selection; (iii) regular and irregular eutectic and peritectic microstructures; (iv) effects of convection on microstructure formation; (v) solidification at a high volume fraction of solid and the related formation of pores and hot cracks; and (vi) solid-state transformations as far as they relate to solidification models and techniques. In light of this progress, critical issues that point to directions for future research in both solidification and solid-state transformations are identified.

(C) 2008 Acta Materialia Inc. Published by Elsevier Ltd. All rights reserved.
\end{abstract}

Keywords: Solidification microstructures; Solid-state phase transformations; Solid-liquid interface; Phase-field modeling; Atomistic modeling

\section{Introduction}

The basic problem of understanding and controlling microstructures during solidification continues to present numerous scientific and technological challenges. Reviews from two workshops, held at Zermatt in 1988 and 1998, have been published in this journal reflecting the advances in solidification science at the end of the 20th century [1,2]. The last workshop of this kind took place in Ames, IA, in 2006. Its main sessions focused on areas of solidification that have been extensively studied by one of the coauthors (R.T.) during the past 40 years. During this period, solidi-

\footnotetext{
* Corresponding author. Tel.: +41 21693 2982; fax: +41 216935890.

E-mail address: wilfried.kurz@epfl.ch (W. Kurz).
}

fication science advanced considerably through pioneering studies of segregation, morphological stability and microstructure selection, and, more recently, through rapid developments in both experimental and multiscale computational tools that have yielded a wealth of new information and quantitative insights into dendritic and twophase microstructures in a variety of alloys. The 2006 Ames workshop distinguished itself from the previous Zermatt workshops by the inclusion of one session devoted to exploring parallels between solid-state phase transformations and solidification. This session highlighted the potential for cross-fertilization of ideas and techniques between these two fields.

The dual goal of this review, which is based on the presentations of the Ames workshop and on research of the 
following two years, is to describe recent developments in solidification science, as they relate to the formation and control of solidification microstructure in metallic materials that point to interesting future directions, and to discuss certain solid-state parallels.

Section 2 is devoted to reviewing recent progress made in using atomic-scale simulations to determine fundamental equilibrium and non-equilibrium properties of the solid-liquid interface. Those include the excess free energy of the solid-liquid interface and the interface attachment kinetic coefficient, as well as their anisotropies, and other parameters that characterize the departure from chemical equilibrium at the interface. Even though those parameters form the basis of all microstructure modeling, they have been notoriously difficult to measure experimentally. Insight into the difficult topic of nucleation can also be obtained by the use of atomistic modeling. From this standpoint, these techniques have provided an unprecedented opportunity to determine the behavior of metallic systems and have emerged as indispensible new tools in solidification science.

Section 3 discusses insights in pattern selection obtained using the phase-field method that has emerged as a method of choice for quantitative simulations of interface dynamics. Dendrite growth at small growth rates is discussed, with particular emphasis on the question of orientation selection. Anomalous dendrite growth directions with off crystal axes are explained in the setting of an enlarged two-dimensional parameter space characterizing the anisotropy of the interfacial free energy derived from atomistic simulations. Rapid dendrite growth and associated transitions in the solidification front morphology are also discussed.

In Section 4 results of experiments and phase-field computations of coupled growth of eutectic and peritectic alloys are presented. Recent theoretical and experimental results of morphological stability and spacing selection mechanisms of the two-phase interfaces are shown. Industrially important irregular eutectic growth, which is controlled by anisotropic interface properties, is also considered.

Section 5 concerns the effect of convection on solidification microstructure. Coupling of macroscopic fluid flow with microscopic temperature and solute fields is instrumental for a better understanding of practically relevant situations where convection always plays an important role. The influence of convection on columnar and equiaxed dendritic growth, on dendrite trunk/arm development, on the corresponding spacings, and on eutectics and peritectics is presented.

Solidification under industrial conditions often involves large thermal gradients and thus induces thermal strains and residual stresses in the final product. This has many practical consequences that engineers must account for. In this context, Section 6 is concerned with solidification microstructures at high solid fractions and related defects, such as pores and hot cracks. Understanding such defects requires a combined treatment of heat and mass transport and of deformation, including nucleation and growth of the voids, as well as percolation of the solid network.

Once the primary process of solidification is complete, the solid generally transforms into new phases and secondary microstructures. As discussed in Section 7, solidification models and related experimental techniques such as directional solidification provide a valuable basis to examine and understand various transformations in the solid state. On the other hand, solid-state transformations provide an interesting setting to test these models in new regimes that are not usually accessible in a solidification context, such as the limit of absolute morphological stability at high growth rates.

We conclude each of the above sections with an outlook statement that is intended to identify the most critical outstanding questions, along with promising areas of future research. It is hoped that this review will stimulate future research to complete our picture of these important and interesting phenomena.

\section{Atomistics of the solid-liquid interface}

In phase transformations the evolution of microstructure is influenced both by transport phenomena and the formation of heterophase interfaces. In this section we consider the second class of processes, as the related interfacial properties, which are not well known, influence all solidification phenomena. In solidification, atomistic processes at the solid-liquid interface are typically described with reference to three parameters: (i) the interfacial free energy, $\gamma$, representing the reversible work to form an interface between solid and liquid phases; (ii) the kinetic coefficient, $\mu$, reflecting attachment kinetics; and (iii) the velocitydependent partition coefficient, $k(V)=x_{\mathrm{S}} / x_{\mathrm{L}}$, with $x_{\mathrm{S}}$ and $x_{\mathrm{L}}$ denoting solute mole fractions on solid and liquid sides of the interface (see the list of symbols in the appendix). Sharp-interface theories and phase-field simulations have demonstrated pronounced sensitivities of solidification rates and growth morphologies to the magnitudes and crystalline anisotropies of these interfacial properties (see e.g. [3-5] and Section 3).

While the availability of accurate values for these interfacial parameters is thus critical for quantitative modeling of solidification microstructures, significant challenges are associated with their direct measurement. To date, limited measurements have been reported for $\gamma$ anisotropy in alloys [6-9]. The magnitude of $\mu$ in metals has been measured directly for only two elemental systems $[10,11]$, and experimental data for the anisotropy of $\mu$ or $k(V)$ in such systems is lacking. This situation has provided strong motivation for the application of atomic-scale simulations as a framework for the direct calculation of both equilibrium and non-equilibrium properties of crystal-melt interfaces in metals, alloys and related model systems [12-14]. This section reviews these results, derived within the framework of molecular dynamics (MD) methods (an introduction to MD methods can be found in Ref. [15]). 
Owing to the required system sizes and time scales, the MD simulations discussed here are based on the use of classical models for interatomic forces. Studies of crystalmelt interface properties have made use of both idealized pair-potential models, such as Lennard-Jones (LJ), and the more realistic many-body embedded atom method (EAM) potentials for metals [16,17]. Comparisons of results obtained with different potential models has proven useful in determining the extent to which specific interface properties are sensitive to the details of the interatomic interactions, or are dictated more generally by factors such as the crystal structure.

In the following the topic is presented in three parts: solid-liquid interface energies and their anisotropies, interface kinetics and recent applications of atomistic simulations in the investigation of crystal nucleation.

\subsection{Crystal-melt interfacial free energies}

In the formation of solidification microstructures, both the magnitude of $\gamma$ and its crystalline anisotropy play important roles. The former represents a critical term in the reversible work to form a solid nucleus, and thus has a strong influence of nucleation kinetics. Further, significant structural variations in $\gamma$ can lead to the primary nucleation of metastable phases in deeply undercooled melts. The anisotropy of $\gamma$ accounts for a sizeable contribution to the interface stiffness, which underlies GibbsThomson effects for anisotropic interfaces, and can thus be an important factor governing growth morphologies (see Section 3 below). We review here the current state of understanding of both the magnitude and anisotropy of $\gamma$ in metals in light of experimental observations and atomistic-simulation results.

\subsubsection{Magnitudes of crystal-melt interfacial free energies}

In the seminal work of Turnbull [18], the first measurements of $\gamma$ were derived over 50 years ago from nucleation studies in undercooled melts. Turnbull demonstrated a strong correlation between $\gamma$ and the ratio of the latent heat of melting ( $L$, per atom) to the average interfacial area per atom (expressed as $\rho^{-2 / 3}$, where $\rho$ is the solid atomic density):

$\gamma=\alpha L \rho^{2 / 3}$

In Eq. (1) $\alpha$ is a constant that has come to be referred to as the Turnbull coefficient; it was originally reported to have a value of approximately 0.45 for metals, and this value describes well the values of $\gamma$ compiled from more recent nucleation data in Ref. [19]. Slightly higher estimates are derived from data based on measurements other than nucleation: $\alpha=0.6$ was quoted [20] for face-centered cubic (fcc) metals based on data [21] from the dihedral-angle technique [22,23], and $\alpha=0.55(8)$ is obtained from analysis considering a combination of measurements in Ref. [24].

In the theory of crystal-melt interfacial free energies due to Spaepen, Meyer and Thompson (SMT) [25-27] the mag- nitude of $\gamma$ is assumed to originate from the (negative) excess entropy associated with the ordering of the liquid against the solid. In this theory the Turnbull relation holds exactly and, under the assumption that the liquid structure is characterized by tetrahedral packing, a number of $\alpha=0.86$ is derived for fcc crystals. This value has been widely applied in modeling of experimental nucleation data [28]. The SMT theory leads to an important prediction that $\alpha$ varies significantly with crystal structure. Specifically, the SMT theory predicts that the Turnbull coefficient for bodycentered cubic (bcc) crystals has a value 18\% lower than that for fcc crystals [25-27]. The lower bcc value is qualitatively consistent with experiments in Fe- and Ni-based systems, where primary nucleation of metastable bcc phases has been observed in rapidly quenched alloys with stable fcc structures [29-36]. The SMT theory was recently employed in calculations of $\alpha$ for a wider variety of compounds, possessing complex "polytetrahedral" order [3739]. These calculations yield results pointing to an even stronger dependence of $\alpha$ on crystal structure. Calculations for the Frank-Kasper $(\sigma)$ phase, the $\lambda$ and $\mu$ phases in Al$\mathrm{Fe}$, and a quasicrystalline icosahedral $(I)$ structure yield: $\alpha_{\mathrm{I}} / \alpha_{\mathrm{fcc}} \approx 0.40<\alpha_{\sigma} / \alpha_{\mathrm{fcc}} \approx 0.42<\alpha_{\lambda} / \alpha_{\mathrm{fcc}} \approx 0.46<\alpha_{\mu} / \alpha_{\mathrm{fcc}} \approx$ $0.51<\alpha_{\mathrm{bcc}} / \alpha_{\mathrm{fcc}} \approx 0.82$. These results, when combined with classical nucleation theory, are consistent with observations of primary nucleation in maximum undercooling measurements for $\mathrm{Al}-(\mathrm{Cu}) \mathrm{Fe}, \mathrm{Al}-(\mathrm{Cu}-) \mathrm{Co}, \mathrm{Al}-(\mathrm{Ni}-) \mathrm{Co}$ and $\mathrm{Al}-\mathrm{Pd}-\mathrm{Mn}[28,40,41]$.

Since the pioneering work of Broughton and Gilmer [42], atomistic simulations have been applied extensively in calculations of $\gamma$ for a variety of systems [12-14]. Fig. 1 summarizes the results to date from atomistic calculations for metals modeled with EAM potentials [43]. The results are plotted in a manner inspired by Eq. (1), as $\gamma \rho^{-2 / 3}$ vs. $L$, where the slopes of the best-fit lines correspond to the average Turnbull coefficients for fcc and bcc

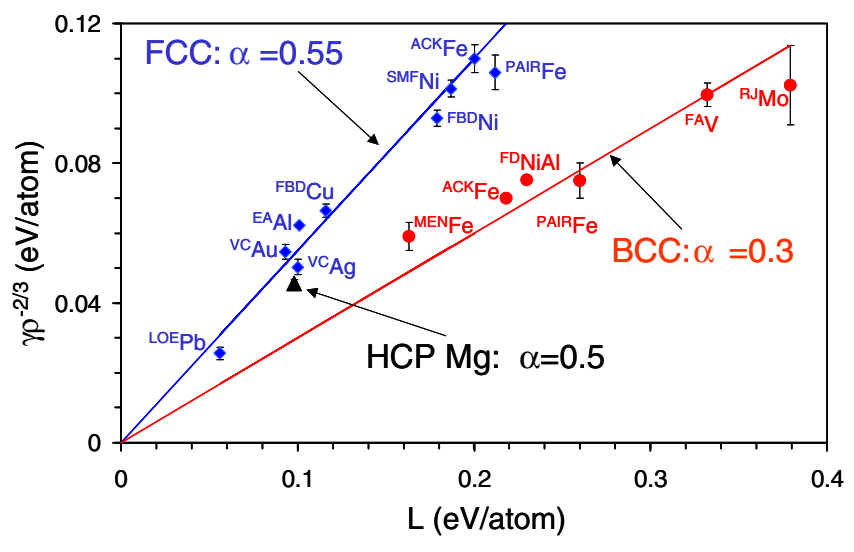

Fig. 1. Calculated interface free energies for metals as a function of latent heat of melting. The superscripts in the labels of the data points correspond to the authors who developed the different interatomic potentials, and are explained in Ref. [12]. Note that for some of the elements (e.g. Fe and Ni) more than one potential was used and, since these potentials generally gave different melting points and latent heats, they led to different estimates for $\gamma$. 
metals (a result is also plotted for hcp $\mathrm{Mg}$ ). The average fcc value of $\alpha=0.55$ derived from the simulations is within the range of the experimental estimates quoted above. The simulation results also confirm the trend towards lower average values of $\alpha$ for bcc relative to fcc structures. In two recent simulation studies [44,52] the crystal-structure dependence of $\alpha$ was considered in detail, employing interatomic potentials that gave rise to both stable and metastable fcc and bcc phases, allowing $\gamma$ to be calculated directly for both crystal structures, with the same underlying potential. For a system with repulsive power-law potentials (of the form $1 / r^{n}$, with $n=6-8$ ), $\alpha$ for bcc was found to be on average about 15-20\% lower than for fcc [52]; simulations for an EAM model of Fe gave values of $\alpha$ for bcc $30-35 \%$ lower than fcc [44]. These results provide direct evidence of a sizeable effect of crystal structure on $\gamma$, and confirm the trend towards a significant reduction in $\alpha$ for bcc structures relative to fcc.

In the plot of calculated interfacial free energies for EAM metals shown in Fig. 1, scatter in the data about the best-fit lines is apparent, particularly for bcc metals. The results highlight the fact that, although the Turnbull scaling relation, with constant $\alpha$ for a given crystal structure, provides a good estimate of the value of $\gamma$, it is not exact. Indeed, atomistic simulations have shown clear variations in $\alpha$ within a given crystal structure, depending on the detailed nature of the interatomic potentials $[52,46]$, and they also have provided some hints into the nature of these dependencies. For repulsive $1 / r^{n}$ potentials one obtains increasing values of $\alpha$ (from 0.47 to 0.60 ) as the range of the interaction decreases (from $n=6$ to $n=\infty$ ) [52]. Similarly, Morris et al. [46] calculated $\gamma$ for fcc Al employing three different EAM potential models, each giving similar values for the melting temperature, $L$ and $\rho$; the potentials gave rise to values of $\alpha$ ranging between 0.41 and 0.60 , and show a trend towards higher interfacial free energies for potentials yielding more ordered liquid structures.

\subsubsection{Crystalline anisotropies}

The importance of $\gamma$ anisotropy for the evolution of solidification microstructures has long been appreciated, particularly in the context of dendritic solidification. In metals and alloys, crystal-melt interfaces are typically rough at the atomic scale, with interfacial free energies that vary smoothly with the orientation of the interface normal $(\hat{n})$. Historically, the orientation dependence of $\gamma$ in cubic metals has been described with a single anisotropy term parameterizing the strength of the fourfold anisotropy (see Section 3). However, atomistic calculations have established that accurate parameterizations of $\gamma(\hat{n})$ for fcc metals generally require two anisotropy parameters associated with both four- and sixfold anisotropy terms:

$\gamma(\hat{n})=\gamma_{o}\left[1+\varepsilon_{1} K_{1}\left(\theta_{1} \varphi\right)+\varepsilon_{2} K_{2}\left(\theta_{1} \varphi\right)\right]$

where $\theta$ and $\phi$ are the spherical angles of the interface normal, $\gamma_{0}$ represents the orientation-averaged interfacial free energy, and $\varepsilon_{1}$ and $\varepsilon_{2}$ parametrize the strength of the four- and sixfold anisotropy cubic-harmonic functions $K_{1}$ and $K_{2}$ [47] (in terms of the components $n_{i}$ interface normal $\hat{n}$, these functions can be written as $K_{1}=\sum_{i=1}^{3} n_{i}^{4}-3 / 5$ and $\left.K_{2}=3 \sum_{i=1}^{3} n_{i}^{4}+66 n_{1}^{2} n_{2}^{2} n_{3}^{2}-17 / 7\right)$.

The need for a two-term anisotropy expansion for $\gamma(\hat{n})$ in fcc metals was first demonstrated in atomistic simulations for EAM Ni [48] and Al [49], employing an MD technique that was specially designed to probe $\gamma$ anisotropies through analyses of equilibrium capillary fluctuations. Calculated results for $\varepsilon_{1}$ and $\varepsilon_{2}$, derived from this capillary fluctuation method (CFM) for a number of other fcc metals and alloys are plotted in Fig. 2, adapted from Ref. [50] (see references therein and Ref. [51] for citations to the original work). In all cases it is found that $\varepsilon_{1}$ is positive, with magnitudes ranging between $3 \%$ and $10 \%$, while $\varepsilon_{2}$ is negative, with values roughly an order of magnitude smaller. Spherical plots of the inverse of the interfacial stiffness Eq. (6), shown in Fig. 2a, illustrate that positive $\varepsilon_{1}$ and negative $\varepsilon_{2}$ values favor dendrite growth directions along $\langle 100\rangle$ and $\langle 110\rangle$, respectively, as discussed in more detail in Section 3.

It is important to emphasize that the nature of $\gamma$ anisotropies calculated for fcc EAM metals have also been found in a wide variety of model fcc systems, including hard spheres [45], repulsive power-law [52] and LJ potentials [53,54]. Additionally, these findings have been obtained both by the CFM and an independent MD method involving interface cleaving [42,54,55]; the results are thus robust with respect to the details of the calculations. All of the calculated results for EAM and model systems are consistent with the ordering $\gamma_{100}>\gamma_{110}>\gamma_{111}$ for the high-symmetry $\{100\},\left\{\begin{array}{lll}1 & 1 & 0\end{array}\right\}$ and $\left\{\begin{array}{lll}1 & 1 & 1\end{array}\right\}$ planes; differences between $\gamma$ for these orientations are generally found to be on the order of a few percent. This relative ordering of the magnitudes of $\gamma$ values, as well as the signs of the calculated anisotropy parameters in Fig. 2, are consistent with experimental equilibrium-shape measurements for Al alloys [6-8]. The fact that all fcc systems modeled to date have been found to yield positive values for $\varepsilon_{1}$ and negative (or statistically insignificant) values of $\varepsilon_{2}$, respectively, for a wide variety of interaction potentials suggests that this feature of fcc $\gamma$ anisotropy, and the inherent competition between $\langle 100\rangle$ and $\langle 110\rangle$ dendrite growth it implies, are intrinsic features dictated primarily by the symmetry of the crystal.

Solvability theory and phase-field simulations [50] (see below) have established that, in the space of $\varepsilon_{1}$ vs. $\varepsilon_{2}$ shown in Fig. 2, dendrite growth along the commonly observed $\langle 100\rangle$ crystallographic directions is stable for large values of $\varepsilon_{1}$, while large and negative values of $\varepsilon_{2}$ tend to favor $\langle 110\rangle$ growth, with a region of "hyperbranched" structures in between (see Section 3). Additions of $\mathrm{Zn}$ to Al have been shown to lead to a transition from $\langle 100\rangle$ to $\langle 110\rangle$ growth with increasing $\mathrm{Zn}$ concentration, with hyperbranched morphologies at intermediate compositions [56], and these results have been interpreted to originate from concentration dependence of $\gamma$ anisotropies. Recent MD calculations [51] for a model LJ alloy system support the assumption 
a

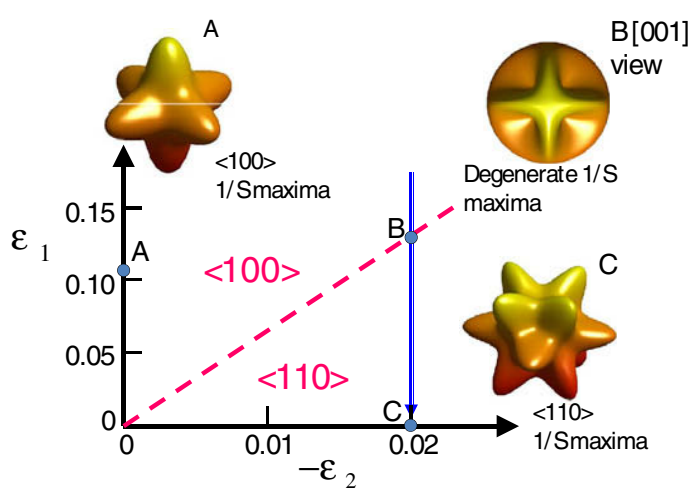

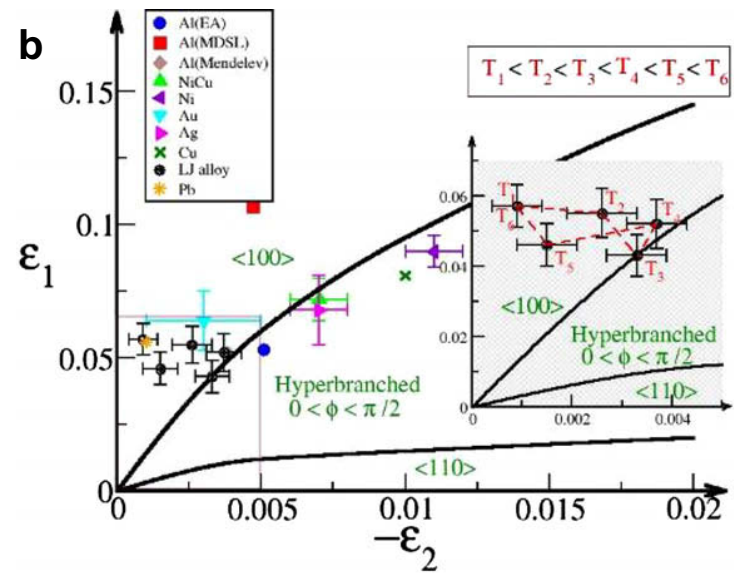

Fig. 2. Orientation selection maps (a) according to the minimum stiffness criterion in the plane ( $\left.\varepsilon_{1}, \varepsilon_{2}\right)$ of anisotropy parameters and illustrative spherical plots of inverse stiffness for different values of these parameters. (b) Dendrite growth directions as a function of $\varepsilon_{1}$ and $\varepsilon_{2}$. The symbols correspond to atomistic calculations of $\gamma$-anisotropy parameters $\varepsilon_{1}$ and $\varepsilon_{2}$ obtained for fcc metals and alloys. The region denoted by thin solid lines in the lower left is shown in more detail in the inset on the right. Adapted from Haxhimali et al. [50].

that solute effects can induce significant changes in $\gamma$ anisotropies. The calculated $\mathrm{LJ}$ results at six temperatures are shown in the inset to Fig. 2 ; anisotropies at $T_{1}$ and $T_{6}$ correspond to the constituent pure materials (with the same anisotropies in a LJ model), while the most concentrated alloy compositions correspond to $T_{3}$ and $T_{4}$. The calculated concentration dependencies for $\varepsilon_{1}$ and $\varepsilon_{2}$ are seen to be significant on the scale required to induce changes in dendrite growth orientations.

\subsection{Interface kinetic properties}

In the solidification of a pure material, the interface kinetic coefficient $(\mu=V / \Delta T)$ relates the degree of interface undercooling $(\Delta T)$ to the interface velocity $(V)$. In materials where significant structural rearrangement is required for crystallization from the melt, growth is generally described within a "diffusion limited" model (e.g. [57$60]$ ), where it is assumed that the activation energy for atom attachment to the crystal face is comparable to that for diffusion in the liquid phase. This model leads to an expression for $\mu$ proportional to the liquid diffusivity $(D)$. Turnbull and Bagley [61] noted that solidification in pure metals can occur at very high undercoolings, where $D$ is very small, and thus growth cannot be diffusion limited. Rather, the frequency of liquid-atom "collisions" with the crystal face was suggested to be the rate-limiting factor. An upper bound for $\mu$ within this collision-limited growth model is given as [62]:

$\mu<\frac{V_{\mathrm{s}} L}{k_{\mathrm{B}} T_{\mathrm{M}}^{2}}$

where $V_{\mathrm{s}}$ denotes the sound velocity and $T_{\mathrm{M}}$ is the melting temperature. For elemental fcc systems results of atomistic simulations have led to refinements in the estimated magnitudes of $\mu$, and have led to the identification of significant crystalline anisotropies in this kinetic parameter.
In the first application of atomistic simulations to the study of crystal-melt interface kinetics, Broughton, Gilmer and Jackson (BGJ) [63] also concluded that the high interface velocities obtained in MD at low undercoolings could not be explained within a diffusion limited growth model. The authors proposed a growth law that fit well their MD data, and implies a form for the kinetic coefficient given as:

$\mu=C \frac{V_{\mathrm{T}} L}{k_{\mathrm{B}} T_{\mathrm{M}}^{2}}$

where $V_{\mathrm{T}}=\sqrt{K_{\mathrm{B}} T / M}$ is the thermal velocity, $M$ is the atomic mass and $C$ is a constant with a value of 1.2 derived from a fit to the MD data for the (100)-oriented LJ interface considered by BGJ. Subsequent to the work of BGJ, experimental measurements of $\mu$ were performed by Rodway and Hunt [11] using the Seeback effect to measure the temperature of solidifying/melting interfaces; values of $\mu=28(8) \mathrm{cm} \mathrm{s}^{-1} \mathrm{~K}^{-1}$ and $33(6) \mathrm{cm} \mathrm{s}^{-1} \mathrm{~K}^{-1}$ were reported from experiments for melting and growth, respectively. These values were found to agree (within experimental error) with Eq. (3) using the BGJ value for C. Importantly, Eq. (3) gives an estimate for $\mathrm{Pb}$ that is roughly 6 times larger than the measured value. The fact that Eq. (3) can lead to a significant overestimation of $\mu$ in metals was pointed out originally by Hoyt et al. [64] who reported MD calculated kinetic coefficients for $\mathrm{Cu}$ and $\mathrm{Ni}$ that are 4-5 times smaller than this upper bound.

The BGJ simulations and $\mathrm{Pb}$ measurements motivated the development of a classical density-functional theory (DFT) for $\mu$ by Mikeev and Chernov (MC) $[65,66]$. The $\mathrm{MC}$ theory yields an expression for $\mu$ of the form of Eq. (4), with a value of $C=0.72$ for $\{100\}$ interfaces, in reasonable agreement with the BGJ value. An important prediction of the MC theory is that $C$ shows sizeable crystalline anisotropy, with $\mu_{100}>\mu_{110} \sim \mu_{111}$, and $\mu_{100} /$ $\mu_{110}=1.4$. Mikheev and Chernov noted that their pre- 
dicted anisotropy between $\{100\}$ and $\{1111\}$ interfaces was consistent with previous LJ MD simulation results by Burke et al. [67]. As discussed in Section 3, kinetic anisotropy of this magnitude has important implications for dendrite growth at high undercoolings.

Over the past decade MD simulations have been applied extensively to simulations of kinetic coefficients for LJ systems [68-70], hard-sphere [71] and EAM metals [43]. The most recent LJ simulations have been based on simulation approaches distinct from those employed by BGJ (for a review of such methods see [72]), yet they lead to consistent results. In recent simulations for hard spheres [71] $\mu$ for $\{100\},\{110\}$ and $\{111\}$ interfaces were calculated to be 1.44(7), 1.10(5) and 0.64(4), respectively, in the hard-sphere units of $\sqrt{K_{\mathrm{B}} / M T_{\mathrm{M}}}$; given that the entropy of melting for hard spheres is $L / T_{\mathrm{M}}=1.17 \mathrm{k}_{\mathrm{B}}$ [73], these results imply a value of $C=1.23(6)$ for $\{100\}$ interfaces, in excellent agreement with the BGJ result. In Ref. [71], available MD results for EAM systems were scaled into hard-sphere units, yielding values ranging between 0.84 and 1.47 for $\{100\}$ interfaces; when these values are divided by the entropy of melting, they imply values for $C$ in Eq. (3) ranging between 0.8 and 1.4. Thus, the EAM values vary by nearly a factor of 2, depending on the details of the EAM potential and MD method used. However, the averaged values obtained in all MD studies to date are generally consistent with the predictions of Eq. (3), with the BGJ and hard-sphere value of $C \sim 1.2$ providing a good "rule of thumb" estimate for $\{100\}$ interfaces.

Concerning the magnitudes of the kinetic anisotropies, LJ simulations by Huitema et al. [70] yield values of $\mu_{100} /$ $\mu_{110}=1.53$ and $\mu_{100} / \mu_{111}=2.00$. For hard spheres the corresponding values are 1.31(9) and 2.25(18), respectively [71]. Comparable anisotropies have been found for all of the fcc EAM systems studied to date by MD [43,71], with $\mu_{100} / \mu_{110}$ ranging between 1.25 and 1.71 , and $\mu_{100} / \mu_{111}$ ranging between 1.2 and 2.7. For all fcc systems studied by atomistic simulations to date it has been found that $\mu_{100}>\mu_{110}>\mu_{111}$.

\subsection{Atomistic studies of crystal nucleation}

Nucleation processes play a critical role in the formation and evolution of solidification microstructures, particularly in the context of phase selection and grain refinement $[28,74-76]$. Over the past decade atomistic simulations have been applied extensively in studies of both homogeneous and heterogeneous crystal nucleation from the melt. In this section we very briefly review these recent applications, as well as parallel theoretical developments. The intent is not to provide a comprehensive review of the topic of crystal nucleation, but rather to highlight the expanding role that atomistic simulations are having as a tool for gaining insights into phenomena relevant for solidification microstructures.

Recent atomistic simulations of homogeneous nucleation have involved explicit calculations of the nucleation barriers [77-80] and nucleation kinetics [80-82], including non-steady-state effects [83,84]. Central issues addressed in these studies have included the diffuse nature and nonspherical geometries of the crystal nuclei, particularly at high undercoolings. Within the framework of classical nucleation theory quantitative predictions of nucleation rates derived from MD require accounting for the temperature and curvature dependence of the interfacial free energy $(\gamma)$ (e.g. $[85,86])$. The theoretical considerations discussed in Section 2.1 suggest that the magnitude of $\gamma$ should generally increase with increasing temperature (i.e. the excess entropy is negative). This dependence has been demonstrated explicitly in recent MD simulations and their analyses [83,87], although different detailed forms for this dependence have been proposed.

The results of atomistic simulations, and nucleation studies in model colloidal systems [88], have motivated efforts aimed at the development of refined classical theories for homogenous nucleation (see Ref. [85], and references therein). Additionally, diffuse-interface (phase-field) models of crystal nucleation (see Refs. [85,89-92] and references therein) have been extensively developed over the past decade and have been shown to predict the magnitude of nucleation barriers in good agreement with atomisticsimulation results for hard-sphere and LJ model systems. These theories automatically account for the diffuse nature of the solid-liquid interface and give rise to temperaturedependent interfacial free energies, in good overall agreement with atomistic-simulation results.

Recently, MD simulations have also been applied in studies of phase selection in undercooled melts. Primary nucleation of metastable bcc crystals in systems with stable fcc phases have been demonstrated in simulation studies of charged-colloid and LJ systems [82,93-95]; interestingly, no such observations have been reported for systems with either hard-sphere or EAM-type potentials for metals $[78,88,96]$. In the past few years the first MD simulations of heterogeneous nucleation have also been reported. Techniques have been demonstrated for calculating the nucleation barrier and contact angles related to crystal nucleation on external "seed" particles [95,97]. In these simulations the seeds are typically introduced as small crystallites with variable structure and lattice spacing; it has been demonstrated that the strongest reduction in the nucleation barrier is obtained when the seeds are isostructural with the nucleating solid. Further, it has also been shown that when the seeds have a different structure than the stable crystal they can enhance tendencies for forming metastable bec phases.

\subsection{Outlook: atomistics of the solid-liquid interface}

It is anticipated that atomistic simulations will continue to lead to new insights into the properties of crystal-melt interfaces relevant to solidification microstructures. Atomistic simulations provide a framework for performing "numerical experiments" to gain insights into atomistic 
mechanisms and the relative importance of "chemical" interactions, atomic size mismatch and crystal structure in governing interfacial properties. Ultimately, these insights should impact development of refined theories and scaling relations for both equilibrium and non-equilibrium interfacial parameters; suggested areas for future such applications are summarized below. Applications of atomistic methods for direct calculations of interfacial parameters for larger-scale models of specific systems can also be expected to continue, and such applications for alloys will require significant efforts at the development of more quantitative interatomic potential models.

Further work is required to investigate composition effects on $\gamma$. To date only one atomistic study has addressed the concentration dependence of the magnitude of $\gamma$ [51] in a model system, featuring nearly ideal solution thermodynamic properties and zero size mismatch. Studies of systems with more complex chemical interactions and size effects are needed to understand the extent to which such factors affect $\gamma$. In terms of interface kinetics, further MD simulations are required to further elucidate the large range of scaled values obtained for EAM systems. Also, while the thermal-velocity temperature dependence of $\mu$ implied by the BGJ model and hard-sphere scaling arguments has been reported to be consistent with many MD studies, a recent calculation for EAM Ni and Fe suggested the importance of interstitial-like diffusion mechanisms at high undercoolings [98]; such findings should be further investigated. Kinetic simulations for alloys will also be useful as a framework for assessing the accuracy of alternative theories of solute trapping [59,60,99-105]. Such studies should also include investigations of the nature of the anisotropies in alloy kinetic coefficients. Recent work along these lines for the congruent solidification can be found in Ref. [106]; further, such simulations would provide useful benchmarks for future developments of sharp-interface kinetic theories, and extensions of classical DFT models to include solute effects.

The ability to observe the formation of metastable phases and heterogenous nucleation in atomistic simulations raises many interesting possibilities for future work. To make contact with experimental data derived in levitated samples (see reviews in Refs. $[28,41,76]$ ), studies with more realistic potential models for metals would be of interest; the investigation of the correlation between the nature of the structural order in deeply undercooled melts and tendencies related to phase selection in nucleation would be particularly interesting. In the context of heterogeneous nucleation, the simulation tools that have been recently demonstrated provide a framework to make contact with the free-growth theory that has been developed in the context of grain refinement in Al alloys [75]. Central questions that could be addressed with simulations surround the nature of the chemical and geometrical features of seed particles that can induce potent catalysis of crystal nucleation.
Finally, it is expected that atomistic and first-principles methods will play an increasingly important role for calculating properties of solid-solid heterophase interfaces in the context of solid-state transformations. Calculations of anisotropic interfacial energies and free energies for coherent and semi-coherent solid-solid interfaces have been demonstrated [107-109] within the first-principles framework of electronic density-functional theory (an introduction to these methods can be found in Ref. [110]). The integration of these techniques with phase-field methods (e.g. [109]) offers exciting possibilities to address outstanding issues in solid-state phase transformations, as described in Section 7 below.

\section{Pattern selection in dendritic growth}

During solidification of a melt, the crystal appears with different morphologies leading, at the end of the transformation, to a certain microstructure. The latter is at the origin of the properties of the final product, hence its importance in the processing chain. Morphologies or patterns, e.g. cells $[111,112]$ and dendrites, are shaped by the complex dynamical evolution of the solid-liquid interface and are both intricate and varied. Fundamentally understanding which pattern is selected under given growth conditions has been a challenge in solidification science for well over half a century. During the last decade computationally efficient phase-field models [5,113-115] and algorithms [116-118], in turn, have reached a mature stage where they can be used to simulate interface dynamics on experimentally relevant length and time scales $[114,115,119,120]$. Advances in the atomistic simulation methods reviewed in the last section have made it uniquely possible to predict key parameters that control the solid-liquid interface dynamics at both low and high growth rate.

This section concentrates on the major progress made with respect to dendrite theory, i.e. on the selection of tip velocity, dendrite shape and orientation.

\subsection{Dendrite velocity and shape selection}

\subsubsection{Low velocity}

The advent of solvability theory during the last two decades of the last century $[3,4,121,122]$ led to the understanding that the stability parameter

$\sigma=\frac{2 D d_{0}}{R^{2} V}$

which controls the operating state of the dendrite tip is determined by the anisotropy of the interfacial free energy $\gamma$ in the low-velocity regime where the solid-liquid interface is in quasi-thermodynamic equilibrium. Here $R$ is the dendrite tip radius, $V$ is the dendrite growth rate, $D$ is either the thermal or solutal diffusivity, depending on whether the solidification of a pure undercooled melt or the isothermal solidification of an alloy is considered, and, concomitantly, $d_{0}$ is either the thermal or chemical capillary 
length. The Ivantsov transport relation determines the Peclet number $P=R V /(2 D)$ as a function of the tip undercooling or supersaturation, while solvability theory predicts $\sigma$ in terms of the $\gamma$ anisotropy [121]. With rare exceptions [123], analytical and computational studies of dendrite growth have traditionally assumed that this anisotropy can be characterized by only one parameter, traditionally denoted by $\varepsilon_{4}$, which measures the magnitude of the fourfold variation of $\gamma, \gamma=\gamma_{0}\left(1+\varepsilon_{4} \cos 4 \theta\right)$, for materials with an underlying cubic symmetry.

By now, predictions of this theory have been largely validated quantitatively by phase-field simulations in two and three dimensions $[5,116]$. Comparisons with experiments, however, have been few. One reason is that anisotropy is difficult to measure experimentally in metallic systems, although progress in this direction has been made recently [6-8]. Liu et al. [124] examined an $\mathrm{Al}-4 \% \mathrm{Cu}$ alloy under conditions of diffusion-controlled solidification. Their measurements show that the dendrite tip selection follows microsolvability theory.

The results of atomistic simulations in metallic systems embodied in Eq. (2) shed light on another reason. Namely, at least two anisotropy parameters, $\varepsilon_{1}$ and $\varepsilon_{2}$, which are the amplitudes of the first two terms in a cubic harmonic expansion of $\gamma(\hat{n})$ in three dimensions, are needed to characterize this anisotropy. Results of solvability theory calculations and phase-field simulations for equiaxed growth in a pure undercooled melt with the form of $\gamma(\hat{n})$ given by Eq. (2) (Fig. 3) show that $\sigma$ depends sensitively on $\varepsilon_{1}$ and $\varepsilon_{2}$ [125]. Thus, a precise determination of both of these parameters is necessary to predict the dendrite tip operating state and to compare theoretical predictions to experiments. Previously, such comparisons have been made by only considering the anisotropy parameter $\varepsilon_{4}=\left(\gamma_{100}-\gamma_{110}\right)$ $/\left(\gamma_{100}+\gamma_{110}\right)$ measured experimentally by examination of equilibrium crystal shapes in $\{100\}$ planes. These measure-

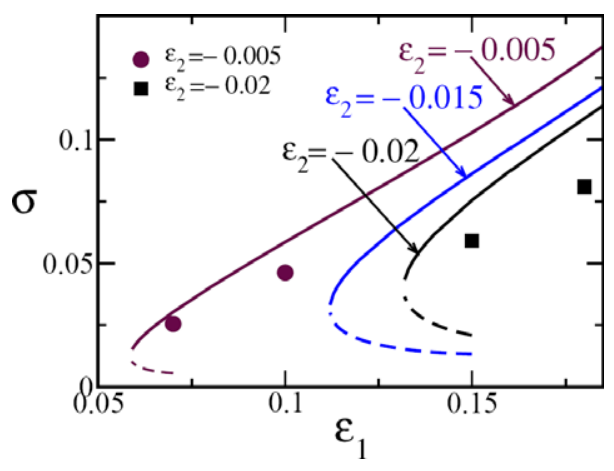

Fig. 3. Dendrite tip selection parameter $\sigma$ as a function of the two crystalline anisotropy parameters $\varepsilon_{1}$ and $\varepsilon_{2}$ for $\langle 100\rangle$ dendrites calculated using solvability theory (solid and dashed lines) and phase-field simulations (symbols) [125]. Solid (dashed) lines correspond to stable (unstable) branches of steady-state dendrite growth solutions. These branches meet at a minimum value $\varepsilon_{1}^{*}$, which shifts further to the right with more negative $\varepsilon_{2}$. The function $\varepsilon_{1}^{*}\left(\varepsilon_{2}\right)$ defines the lower limit of existence of $\langle 100\rangle$ dendrite growth solutions in Fig. 2 and is in good agreement with the $\langle 100\rangle$ hyperbranched boundary determined by phase-field simulations. ments exploit the fact that this shape has the same fourfold variation as $\gamma$ for small $\varepsilon_{4}$. This remains true for the more general form of $\gamma(\hat{n})$, defined by Eq. (2), but the amplitude of the fourfold variation of $\gamma$ (or the equilibrium crystal shape) is given by $\gamma(\hat{n})$. This implies that the experimental measurements of anisotropy to date do not determine $\varepsilon_{1}$ and $\varepsilon_{2}$ independently, as required to predict $\sigma$, but rather determine their combination.

\subsubsection{High velocity}

For large growth rates, in contrast, the departure from thermodynamic equilibrium at the solid-liquid interface strongly influences the selection of the dendrite tip operating state. Phase-field simulations $[119,126]$ of rapid solidification in pure undercooled $\mathrm{Ni}$ have shown that the magnitude and anisotropy of $\mu$ computed by atomistic simulations yield predictions of dendrite growth rates consistent with experimental data. The magnitude of the $\gamma$ anisotropy has been found to have a negligible effect on the tip operating state at these high growth rates and the extension of solvability theory to a kinetically dominated regime [127] has yielded a good agreement with phase-field simulations and experimental data [119].

Two-dimensional phase-field simulations of rapid solidification in highly undercooled pure melts with isotropic interface kinetics have shown the existence of a disordered growth morphology formed by repeated tip splitting at large growth rates [128]. Three-dimensional phase-field simulations that vary the magnitude $\varepsilon_{k}=\left(\mu_{100}-\mu_{110}\right) /$ $\left(\mu_{100}+\mu_{110}\right)$ of the kinetic anisotropy exhibit a similar morphology when $\varepsilon_{k}$ is decreased below a critical value that depends on undercooling [119]. This growth morphology yields a circular envelope of the solidification front, as opposed to an angular envelope for stable dendrite growth without tip splitting. This transition from an angular to a circular envelope with increasing growth rate is distinct from the one associated with absolute stability in rapid alloy solidification $[118,129,130]$ because the interface is still highly unstable in the regime where the envelope is circular. This high-velocity tip-splitting morphology with a circular envelope is also fundamentally different from the seaweed morphology forming in a capillary-dominated growth regime, which has been associated with an underlying steady-state doublon [131] or triplet [132] structure with a split tip in two and three dimensions, respectively. In particular, solvability theory shows that this high-velocity disordered morphology originates from the termination of the main dendrite branch of steady-state growth solutions for undercoolings larger than some threshold that increases with $\varepsilon_{k}[119,127]$. For $\varepsilon_{k} \approx 0.13$ predicted from MD for pure $\mathrm{Ni}$, this threshold is large enough that stable dendrite growth persists to very large undercoolings for pure $\mathrm{Ni}$ or $\mathrm{Cu}$.

\subsection{Dendrite orientation selection}

Metal dendrites typically grow along directions that correspond to low index crystal axes, e.g. $\langle 100\rangle$ directions in 
fcc systems. Perhaps for this reason, the question of how a dendrite selects its growth direction has historically received little attention.

Dendrites, however, sometimes select other directions, "off crystal axes", ranging from early observations of $\langle 2245\rangle$ directions off the basal plane and the $c$-axis in $\mathrm{Mg}$ alloys with hexagonal crystal symmetry $[133,134]$ to $\langle 110\rangle$ and $\langle 111\rangle$ directions for ammonium chloride in aqueous solutions [123], and to more recent detailed observations of $\langle 110\rangle,\langle 320\rangle,\langle 211\rangle$ and unsteady curvilinear dendrite paths in fcc Al-based alloys [135-140]; anomalous growth directions can be of practical interest as in the example of "feathery crystals" in Al alloys that lead to highly anisotropic microstructures with undesirable mechanical properties. These observations have generated renewed interest in the question of how a dendrite selects its growth direction. In turn, the expanded anisotropy parameter space of Eq. (2), derived from atomistic simulations, has set the stage for exploring this question theoretically in the experimentally relevant low-velocity regime where dendrite growth is dominated by capillary anisotropy.

\subsubsection{Extremum stiffness criterion}

Dendrite growth directions have traditionally been assumed to correspond to maxima of $\gamma$. In the simplest two-dimensional situation, where $\gamma=\gamma_{0}\left(1+\varepsilon_{4} \cos 4 \theta\right)$, these maxima also correspond to minima of the interface stiffness, $S=\gamma+\mathrm{d}^{2} \gamma / \mathrm{d} \theta^{2}$, which appears in the Gibbs-Thomson condition. This assumption is physically motivated by the fact that capillary forces are least effective at smoothing out protrusions of the solid-liquid interface in directions where the stiffness is minimum. In three dimensions, the interface stiffness becomes a tensor quantity, but the trace of this tensor for a spherical crystal seed

$S=2 \gamma+\frac{\partial^{2} \gamma}{\partial \theta^{2}}+\frac{1}{\sin ^{2} \theta} \frac{\partial^{2} \gamma}{\partial \varphi^{2}}+\cot \theta \frac{\partial \gamma}{\partial \theta}$

can be used similarly to formulate an extremum criterion for dendrite growth directions $[50,125]$. As in the previous section, $\theta$ and $\varphi$ are the spherical angles of the interface normal. An analytical calculation of the stiffness $S$ defined by Eq. (6) for the form of $\gamma$ defined by Eq. (2) reveals that minima of $S$ (maxima of $1 / S$ in Fig. 2(a)) for cubic crystals correspond either to $\langle 100\rangle$ directions or $\langle 110\rangle$ directions. In the plane of anisotropy parameters of Fig. 2(a), the region of this plane where $\langle 100\rangle$ dendrites are predicted to form lies close to the positive $\varepsilon_{1}$ axis, while the $\langle 110\rangle$ region lies close to the negative $\varepsilon_{2}$ axis. These two regions are separated by a boundary $\left(\varepsilon_{1}=-20 \varepsilon_{2} / 3\right)$ where $S$ is minimum for all directions contained in any of the $\{100\}$ planes. Therefore, using this physically motivated but ad hoc minimum stiffness criterion, one would conclude that dendrites select either $\langle 100\rangle$ or $\langle 110\rangle$ directions and, exactly on the boundary between the $\langle 100\rangle$ and $\langle 110\rangle$ regions, some unspecified directions contained in the set of $\{100\}$ planes, as shown in Fig. 2(a).

\subsubsection{Phase-field simulations and experiments}

Phase-field simulations of dendrite growth in a pure undercooled melt that use the anisotropy form of $\gamma$ defined by Eq. (2) have been carried out to explore the selection of dendrite growth directions [50]. The results shown in Fig. 2 of the atomistic section reveal that $\langle 100\rangle$ and $\langle 110\rangle$ dendrites form near the positive $\varepsilon_{1}$ and negative $\varepsilon_{2}$ axis, respectively, as expected from the minimum stiffness criterion. Not predicted by this criterion, however, is a large region sandwiched between the $\langle 100\rangle$ and $\langle 110\rangle$ regions in Fig. 2 where the dendrite growth directions vary continuously from $\langle 100\rangle$ to $\langle 110\rangle$, as illustrated in the top panel of Fig. 4. Cubic symmetry dictates that these misoriented dendrites have 24 primary branches off crystal axes. These "hyperbranched" structures occupy a large region of
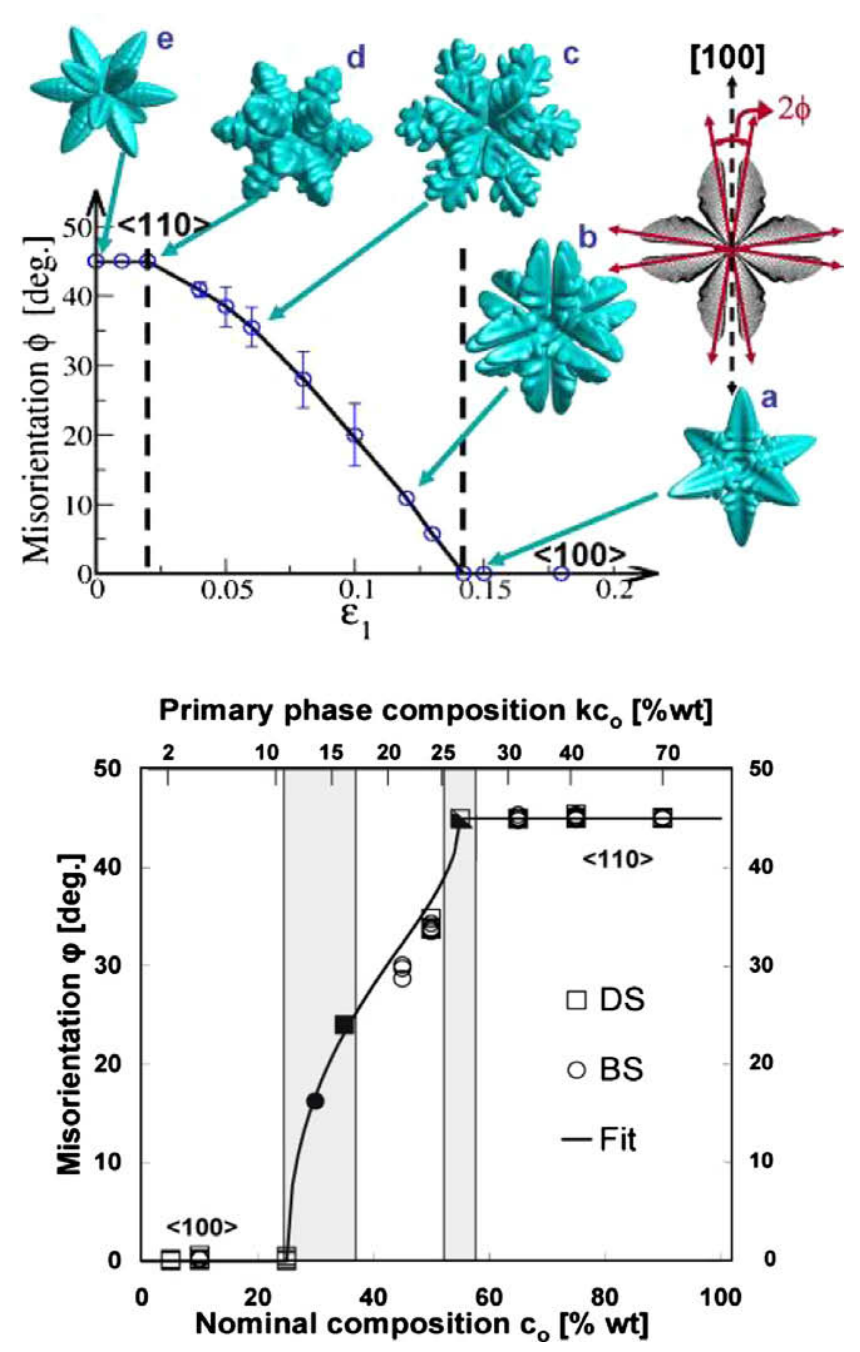

Fig. 4. Phase-field simulations of equiaxed growth (a) and experimental results of dendrite orientation in directionally solidified Al-Zn alloys (b), showing a continuous change of dendrite growth misorientation $\phi$ as a function of anisotropy parameter, $\varepsilon_{1}$, and $\mathrm{Zn}$ composition, respectively [50]. The curve in (b) is an interpolation through the experimental measurements (dots and squares). DS, directionally solidified by chilling, $3>V>0.5 \mathrm{~mm} \mathrm{~s}^{-1}, 3>G>0 \mathrm{~K} \mathrm{~mm}^{-1}$; BS, Bridgman solidification, $V=67 \mu \mathrm{m} \mathrm{s}^{-1}$ and $G=10 \mathrm{~K} \mathrm{~mm}^{-1}$. 
parameter space, while the $\langle 110\rangle$ dendrites are constrained to a narrow wedge above the negative $\varepsilon_{2}$ axis.

The MD data for several pure fcc metals fall strikingly close to the $\langle 100\rangle$-hyperbranched boundary in Fig. 2. This suggests that changes of anisotropy parameters resulting from solute addition are the underlying mechanism for atypical dendrite growth directions observed in fcc metallic alloys [56]. Results of directional experiments in $\mathrm{Al}-\mathrm{Zn}$ alloys that show a continuous change of dendrite misorientation as a function of $\mathrm{Zn}$ composition (bottom panel of Fig. 4) and recent MD simulations in a binary alloy that show that alloying shifts the anisotropy parameters towards the $\langle 100\rangle$-hyperbranched boundary both support this conjecture.

A new experimental technique should also be mentioned, as it allows the use of instant pressure changes to study transient and non-steady-state effects in dendritic growth, particularly in the tip selection process [141]. Theoretical and experimental studies of non-steady-state growth transients have also been carried out that provide new insights into transitions between globular and dendritic microstructures in both equiaxed alloy solidification [142] and semi-solid processing [143]. Related progress in understanding transitions between columnar and equiaxed microstructures is reviewed in Section 5.2.

\subsection{Outlook: pattern selection in dendritic growth}

An accurate quantitative determination of $\gamma$ anisotropy in $\mathrm{Al}-\mathrm{Zn}$ and other alloys using either MD simulations or equilibrium-shape measurements in multiple planes is critically needed to quantify the parameters $\varepsilon_{1}$ and $\varepsilon_{2}$, as well as to investigate whether higher-order cubic harmonics are needed to represent the $\gamma$ anisotropy. In particular, the next higher-order term in the cubic harmonic expansion of $\gamma$ in Eq. (2) generates an eightfold $\cos 8 \theta$ variation in $\{100\}$ planes that can influence orientation selection because the combination of four- and eightfold variations in these planes produces stiffness minima in directions off crystal axes $[56,125]$. In addition to the characterization of crystalline anisotropy, these results bring new challenges for dendritic growth theory. Fig. 3 shows that this theory can predict the limit of existence of $\langle 100\rangle$ dendrites in Fig. 2(b). This theory, however, is presently only developed to predict independently the tip operating state parameter $\sigma$ and the growth misorientation in two dimensions. How to extend this prediction to three dimensions is yet to be elucidated and appears to be a particularly challenging theoretical problem.

Both MD simulations that characterize the departure from chemical equilibrium at the interface and its anisotropy, and three-dimensional phase-field simulations that incorporate this knowledge and simultaneously resolve the thermal and solutal diffusion fields, are still needed. This would lead to the understanding of the fundamental relationships between different rapid solidification phenomena, including the break in the velocity-undercooling relationship, the change from an angular to a spherical envelope of the solidification front below the absolute stability limit and grain refinement at large undercooling.

The growth of partially faceted dendrite growth structures remains comparatively less understood than the growth of non-faceted dendrites reviewed in this section, even though phase-field simulations of faceted growth have started to be carried out $[144,145]$. In addition, recent progress has been made to model the columnar growth of more complex cellular/dendritic array structures during directional solidification using two-dimensional phase-field simulations $[112,146]$. This work identified scaling laws that relate different microstructural length scales, including the primary dendrite array spacing. However, extension of these simulations to three dimensions remains a major challenge even on today's computers. It is also a challenge to model the slow coarsening evolution of dendritic structures in the mushy zone. Recent progress in this direction by a combination of phase-field simulations and experiments by Voorhees and co-workers is reviewed in Section 6.

The study of pattern selection in multicomponent alloys requires further work. Alloy phase-field models can in principle be formulated for an arbitrary number of components, and such models have been used to simulate both eutectic [147] and dendrite growth [148] in ternary alloys. However, formulating models with computationally tractable thin interface limits to achieve quantitative results remains a major challenge for more than two components and/or nonlinear phase coexistence curves. Recent progress to formulate computationally efficient phase-field formulations for binary alloys with nonlinear phase coexistence curves shows promise in this direction [149].

Finally, recent progress has been made to model complex dendritic microstructures in polycrystalline materials using a classical phase-field approach with a scalar order parameter that represents the local grain orientation $[150,151]$. The phase-field crystal (PFC) approach has also recently emerged as a powerful computational approach for multiscale modeling of polycrystalline microstructural evolution [152]. By construct, this method resolves the atomic-scale density wave structure of the crystalline material and can describe its evolution on time scales orders of magnitude longer than molecular dynamics simulations. This method has recently been shown to describe well the equilibrium properties of the crystal-melt interface for the case of pure Fe [153]. In a broader solidification context, it offers a new avenue to tackle complex multiscale problems where crystalline defects play a key role, from the formation of complex structures such as twinned dendrites to grain coalescence during the late stages of solidification.

\section{Pattern selection in coupled growth}

The most widespread solidification microstructures besides dendrites are the composites formed in eutectic 
alloys. In purely metallic (regular) systems the eutectic phases generally form in a coupled manner at the solidification front, which is flat on a scale that is large, compared to the microstructural spacing. In irregular eutectics the growth front is non-isothermal, but a certain coupling does exist. In the last decade, major progress on the understanding of pattern selection in coupled growth has been made thanks to the development of new experimental techniques and efficient phase-field models for multi-phase growth. Here, we concentrate on the influence of tri-junction motion and interfacial properties on pattern selection, and the complex three-dimensional structure of eutectic composites and the associated instabilities and spacing adjustment mechanisms. Microstructures in multicomponent alloys and peritectic coupled growth will also be briefly discussed. This section is divided into regular and irregular growth.

\subsection{Regular eutectic and peritectic growth}

\subsubsection{Stability of coupled growth}

A central question for the understanding of pattern selection in coupled growth is to identify and characterize the dynamic instabilities that limit the range of stable (and hence observable) lamellae or rod spacings [154]. For lamellar growth, new results concerning the instabilities both at small and at large spacings have been established. In a combined experimental and numerical study [155], the lamella elimination instability that occurs at small spacings was investigated in detail. It was found in phase-field simulations that tri-junction points do not move only normal to the growth front, as traditionally assumed [156,157]: they can also slide parallel to the front, as illustrated in Fig. 5(a), with a velocity that is propor- a

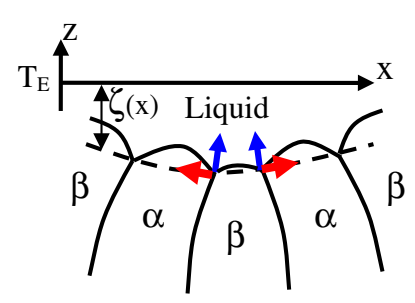

b

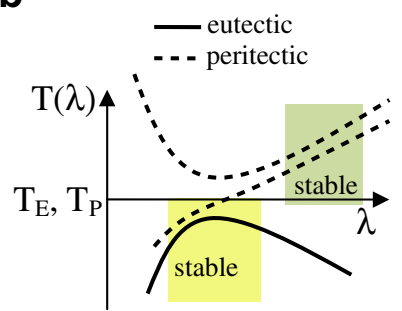

Fig. 5. Schematic illustrations of (a) the sliding motion of three-phase junctions (tri-junctions) and (b) the crucial influence of this motion on the stability of both eutectic and peritectic coupled growth. In (a), the envelope of the composite interface is shown as the dashed line that passes smoothly through tri-junctions. The displacement of this line along the $z$ axis measured from the eutectic temperature (at $z=0$ ) is $\zeta(x)$. The blue arrows depict the motion of the tri-junctions of the central $\beta$ lamella normal to this envelope. Consideration of this motion alone (JacksonHunt-Cahn assumption) leads to the prediction that coupled growth is unstable for $\mathrm{d} T / \mathrm{d} \lambda>0$. The red arrows indicate the lateral motion of the junctions in the direction of increasing spacing. This additional motion can stabilize both eutectic and peritectic coupled growth for a finite range of spacing where $\mathrm{d} T / \mathrm{d} \lambda>0$, as shown in (b) [154]. (For interpretation of color mentioned in this figure the reader is referred to the web version of the article.) tional to the local gradient of spacing [155]. This effect always smoothes out inhomogeneities along the front, such that a lamellar front (in eutectics or in peritectics; see below) can be stable over a limited range of spacing even when the front temperature vs. spacing curve has a positive slope (i.e. $\mathrm{d} T / \mathrm{d} \lambda>0$; Fig. 5(b)), as long as this slope remains below a critical value that increases with the temperature gradient. As a consequence, the critical spacing for the onset of the lamella elimination instability can be substantially smaller than the minimum undercooling spacing, depending on the temperature gradient and the growth speed. Even though the lateral tri-junction sliding is difficult to observe experimentally, the excellent agreement of the stability thresholds between simulations and experiments strongly supports its existence.

\subsubsection{Binary eutectic growth}

Concerning the instabilities at large spacings, major recent advances have come from the study of morphological stability and spacing adjustment mechanisms in "thick" samples which contain a sufficient number of lamellae for the dynamics to be three-dimensional but remain small enough to exhibit only weak convection. For example, directional solidification experiments on the $\mathrm{Al}-\mathrm{Cu}$ eutectic have been carried out in capillary tubes [158], and the evolution of the eutectic spacing was examined by micro-milling and by the reconstruction of the $3 \mathrm{D}$ microstructure [159]. Furthermore, an experimental setup using a long-distance microscope in oblique view was developed to observe the dynamics of growth fronts in bulk samples of transparent alloys in situ [160-162]. In the $\mathrm{Al}-\mathrm{Cu}$ eutectic, it was found that the ratio of the maximum to the minimum spacing was approximately 1.2. In this alloy, a new instability was also discovered: after a sudden change of the growth velocity by a factor of 4 , an initial bump on the lateral surface of a lamella can develop and then grow parallel to the network of lamellae. This mechanism, comparable to dendrite branching (i.e. secondary dendrite arms emitting tertiaries which can then become new primary trunks), reduces the spacing (Fig. 6 [159]). In the transparent alloy, a zigzag instability occurs above a critical spacing [160,161], which leads from straight to wavy lamellar arrays (Fig. 7). Three-dimensional phase-field simulations [163] have confirmed the latter result. In addition, these simulations have shown that the analogues of the oscillatory instabilities known in thin samples do exist, but that they are not observable since they always occur for larger spacings than the zigzag instability.

For rods, only preliminary results on stability are available $[162,164,165]$, and these indicate that the main spacing adjustment mechanisms are rod elimination for small spacings and rod splitting for large spacings, and that the activation of these mechanisms may strongly depend on the geometry of the experiment and the resulting boundary conditions. In both experiments and simulations it was observed that the lamella-to-rod transition is not sharp 


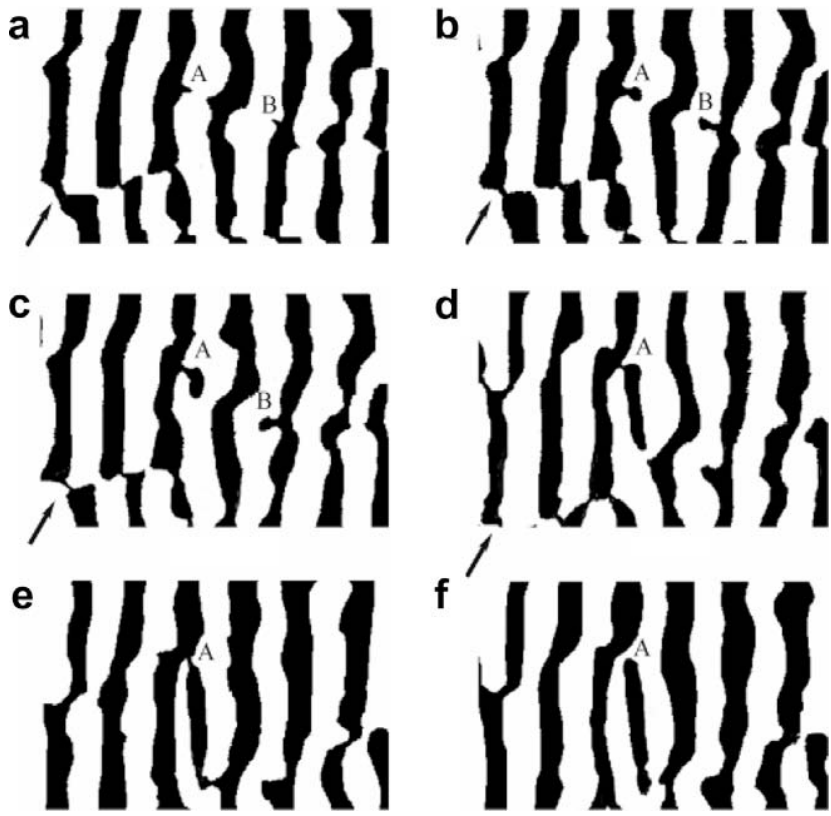

Fig. 6. Successive transverse sections of a directionnally solidified eutectic Al-33.2 wt. \% Cu alloy, showing a mechanism for lamella creation in three dimensions (average eutectic spacing $=4.8 \mu \mathrm{m}$ ). After an increase of the growth speed from $V=1.25$ to $V=5 \mu \mathrm{m} \mathrm{s}^{-1}$, a perturbation normal to the lamella (marked "A") grows and develops into a new lamella which propagates along the pre-existing ones [159]

but occurs gradually over a range of compositions (or volume fractions) [166].

Finally, an investigation of the stability of eutectic growth at high velocities was recently carried out for AlSm alloys [167]. This study showed that, when the temperature dependence of the solute diffusivity in the liquid is taken into account, there exists a maximum undercooling above which a low solubility eutectic will not be stable [168], and this undercooling does not vary with composition. These results are relevant for the eutectic-to-glass transition.

\subsubsection{Multicomponent alloys}

Recently, a whole series of studies has investigated eutectic growth in multicomponent systems. For eutectics with a small amount of ternary impurities, eutectic colonies form. The instability leading to colony formation was found to be similar to the Mullins-Sekerka instability, but with the difference that it can exhibit an oscillatory behavior [147,169]. A clear influence of crystallographic effects on the shape of well-developed colonies has also been reported [169-171]. Ternary-coupled eutectic growth (involving three distinct solid phases) was investigated both in metallic [172] and organic alloys [173-175]. A bewildering variety of microstructures was observed, which opens a completely new field of study. The variety was even greater for quarternary eutectics [176]. There is little recent theoretical work in this area, except a generalization of the Jackson-Hunt calculation to ternary composites [177]. For a more detailed review on solidification in multicomponent alloys, see Ref. [178].

\subsubsection{Peritectic coupled growth}

The question whether peritectic alloys can exhibit eutectic-like coupled growth has been discussed for a long time, but a definite answer has remained elusive. Observations of growth in peritectic systems have remained rather sparse until recently $[179,180]$; rather, mostly other microstructures, such as discrete bands, oscillatory structures and disordered composites, have been found [181-185]. The situation has now been considerably clarified. First, it was established that convection has a strong influence on microstructure formation in peritectic growth (see also Section 5). Secondly, it was recognized that the lateral tri-junction motion discussed above for eutectics is also present in peritectics and can lead to stable coupled growth even when the slope of the front temperature vs. spacing curve is positive, which is often the case in peritectics. Furthermore, a detailed experimental and numerical study of two-phase microstructure selection in the $\mathrm{Fe}-\mathrm{Ni}$ system (Fig. 8 [186,187]) was carried out. The main results are (Fig. 9): (i) stable coupled growth in Fe-Ni can occur only above a critical temperature gradient where both phases are morphologically stable and (ii) the range of stable spacing strongly depends on composition and is limited for both large and small spacings by oscillatory instabilities. Recently, peritectic coupled growth was also observed in the $\mathrm{Cu}-\mathrm{Sn}$ alloy [188].

\subsubsection{Initiation of coupled growth}

A question of great interest is how coupled growth develops from a single solid primary phase. Recent experimental and phase-field modeling studies have shed light on two qualitatively different mechanisms of initiation $[186,188,189]$. The first, which applies to both eutectic
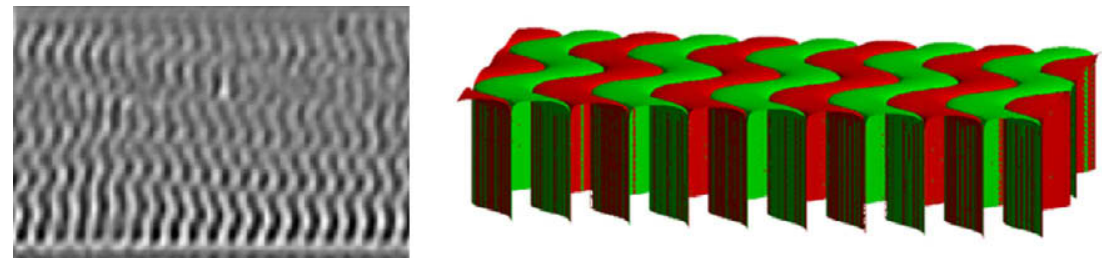

Fig. 7. "Zig-zag" structures in a near-eutectic transparent eutectic alloy $\mathrm{CBr}_{4}-\mathrm{C}_{2} \mathrm{Cl}_{6}$. Left: top view of an in situ experiment using an optical long-distance microscope in oblique view and subsequent image processing, $G=100 \mathrm{~K} \mathrm{~cm}^{-1}, V=0.5 \mu \mathrm{m} \mathrm{s}{ }^{-1}$ (the growth direction is normal to the plane of the page, the average spacing $=20 \mu \mathrm{m}$ ) [161]. Right: snapshot of a 3D phase-field simulation of a similar model system [163]. Growth direction is upward. 
a

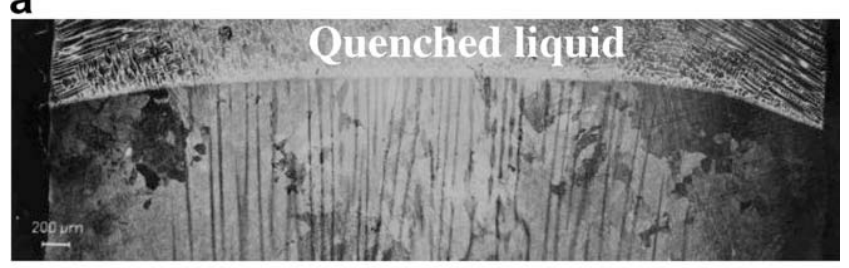

b

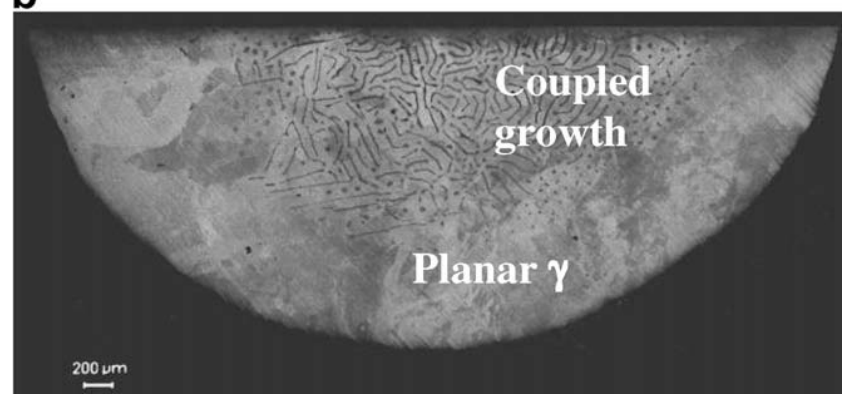

Fig. 8. Peritectic coupled growth of lamellar and fibrous $\delta$ and $\gamma$ in a directionally solidified specimen of $\mathrm{Fe}-4.1$ at. $\% \mathrm{Ni}\left(G=18 \mathrm{~K} \mathrm{~mm}^{-1}\right.$, $V=10 \mu \mathrm{m} \mathrm{s}^{-1}$ ). Longitudinal (a) and transverse (b) micrographs. The microstructure is correlated with the local Ni composition, which increases in radial direction due to macrosegregation [187].

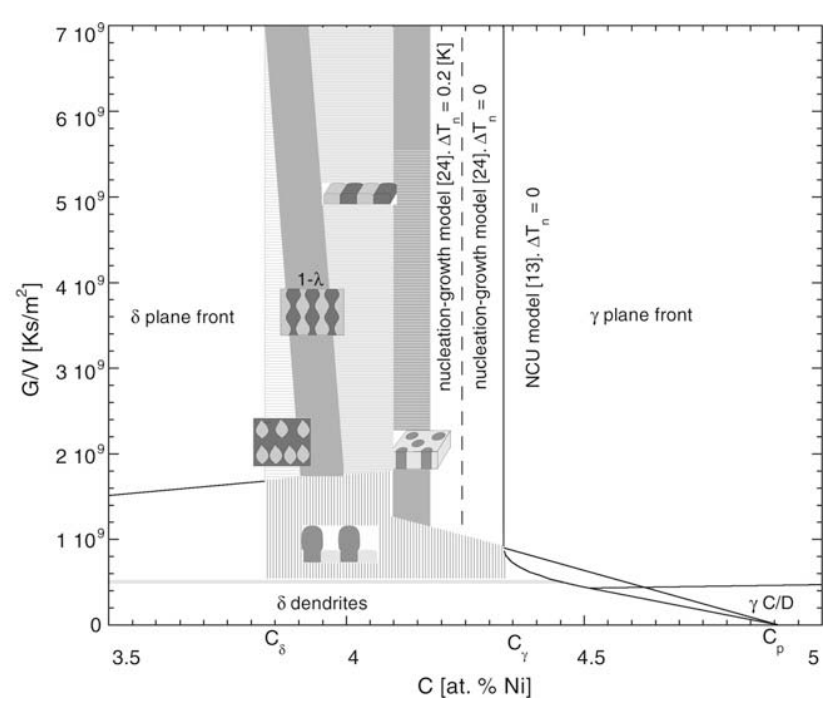

Fig. 9. Microstructure selection map for steady-state directionally solidified peritectic $\mathrm{Fe}-\mathrm{Ni}$ alloys with plane front coupled growth of fibrous and lamellar structures in the center, oscillatory structures on the left and cellular composites at low $G / V$ values [187].

and peritectic alloys, involves a growth instability occurring during spreading of the second phase on the primary phase. This mechanism was studied experimentally in a transparent eutectic alloy in thin samples [189]. After nucleation of the second phase, a "finger" develops which propagates laterally along the solid-liquid interface. Above a certain velocity, it develops an oscillatory instability, which leads to the emergence of very fine lamellae. This is followed by numerous lamella elimination events until a stable state is reached. Similarly, peritectic coupled growth in $\mathrm{Cu}-\mathrm{Sn}$ alloys was initiated by repeated sideways growth of both phases [188]. The second mechanism, which has only been observed so far in the peritectic Fe-Ni system, involves nucleation of the secondary phase at multiple sites on the primary phase. The latter can initiate coupled growth if the spacing between nuclei falls inside the stable range of coupled growth spacing, or an island banding microstructure often seen as a transient leading to coupled growth [186].

\subsection{Irregular eutectic growth}

Irregular eutectic structures are developed when a nonfaceted (nf) phase is coupled with a faceted (f) phase, as observed most notably in the $\mathrm{Fe}-\mathrm{C}$ (graphite) and $\mathrm{Al}-\mathrm{Si}$ systems. In such eutectics, local morphological adjustment of interphase spacing is severely encumbered by the limited branching ability of the highly anisotropic faceted phase containing planar defects. Thus, the spatially non-uniform or irregular structure that evolves during $\mathrm{f}-\mathrm{nf}$ eutectic solidification is inherently three-dimensional, where the relationship between the growth mechanisms of the faceted phase and the complex non-isothermal interface structure gives rise to a more diverse range of solidification microstructures than that exhibited by regular eutectics and to a number of growth mode transitions involving both intrinsic and extrinsic mechanisms of nucleation and growth.

From a theoretical standpoint, only limited progress has been made over the last decade toward understanding the dynamics of irregular eutectic growth, and new developments remain constrained to two-dimensional (2D) descriptions which are inherently limited. Most recently, a fourth-order shape function was used [190] to extend the analytical MK model [191] (which employs a cubic function) to allow for the deformation of the phases in a non-isothermal coupled growth configuration. Accordingly, both the protrusion of the leading (faceted) phase and the depression of the non-faceted phase are parameterized with the assumed shape function and related to the contact angles for the respective phases at the triple junction. While this modification to the MK theory addresses an important feature of the non-isothermal interface, it appears that significant advancement can only arise from more descriptive 3D parameterization of the irregular growth front coupled with models that account for the variation in local structure, the role of crystallography and crystal defects, the simultaneous operation of multiple nucleation and growth mechanisms, and the competition that leads to various observed growth mode transitions.

Focused ion beam tomography has recently been used by Lasagni et al. [192] for high-resolution investigation of the $3 \mathrm{D}$ structure of Sr-modified and unmodified $\mathrm{Al}-\mathrm{Si}$ alloys (Fig. 10), and volume-based structural parameters have been used to identify the flake/fiber transition, which is not indicated through conventional spacing measurements [193]. These efforts to understand the 3D nature of irregular eutectic growth are limited in number, however, 

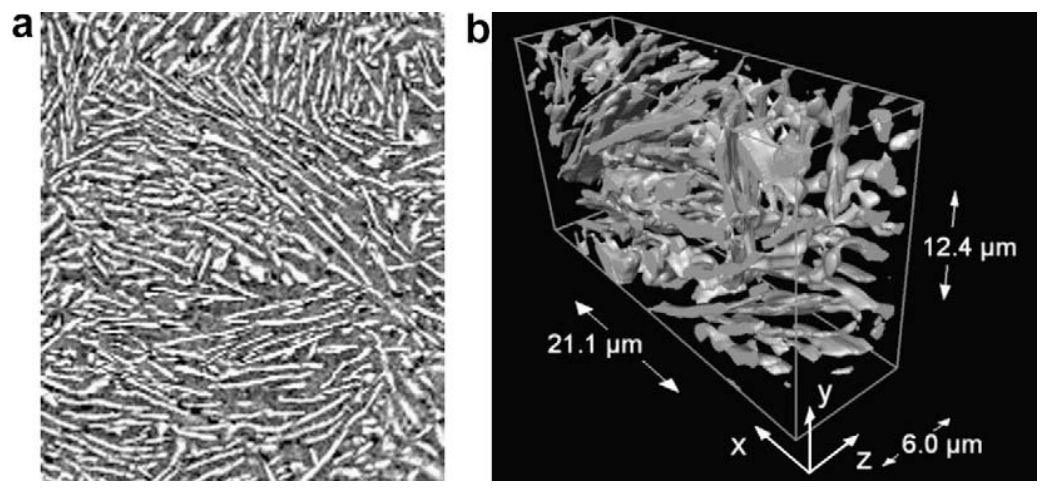

Fig. 10. Microstructure of an unmodified Al-12 wt.\% Si alloy. (a) Metallographic image and (b) 3D reconstruction of the deep-etched Si structure [192].

and several intrinsic and extrinsic growth mode transitions in irregular eutectic systems remain poorly understood.

We focus here on $\mathrm{Al}-\mathrm{Si}$ as the prototypical irregular eutectic alloy and examine recent investigations into intrinsic growth morphologies and impurity effects leading to microstructural modification. Recent investigations of the intrinsic high-velocity flake/fiber (sometimes called quench modification) transition in the binary Al-Si eutectic have indicated that this transition occurs over a rather wide temperature range and in several stages. Observation of inplane fingering of flakes, flake-like packets of rods and out-of-plane rod growth suggest that anisotropic interfacial properties are critical here [193], emphasizing the need for experimental and atomistic determination (see Section 2) of these properties. Low-velocity transitions to angular scriptlike and other morphologies have also been associated with this anisotropy and related to a twin-enhanced growth mechanism [194]. At low velocities, the script-like structure gives way to the strong selection of a $\langle 001\rangle$ textured, faceted and twinned silicon morphology, which can be attributed to the effective kinetic operation of a complex structure of coherent $\{310\}$ and $\{210\}$ twins in the silicon phase [195].

Because the competition between the available mechanisms of nucleation and growth are central to the selection of $\mathrm{f}-\mathrm{nf}$ morphologies, solute elements, even in trace amounts, can play a critical role in altering the observed dynamics. In the case of $\mathrm{Al}-\mathrm{Si}$, recent results bring into question whether the influence of impurities on growth mechanisms can ever be completely avoided in practice. It has been reported [196] that, in addition to the wellknown modification effect, $\mathrm{Sr}$ additions may alter the mechanism by which eutectic grains nucleate. Indeed, three distinct mechanisms for silicon nucleation have been reported, each at different Sr levels [197]. Subsequent findings [198-200] suggest that Sr may poison the catalytic effects of other impurities, thereby suppressing Si nucleation and increasing the eutectic grain size in commercial alloys. It has also been proposed by Shankar et al. [201] that trace amounts of $\mathrm{Fe}(>30 \mathrm{ppm}$, which is commonly observed even in high purity Al) may have a strong influence on eutectic growth morphology. Examining this issue in hypoeutectic Al-Si alloys, they claim that nucleation of
$\mathrm{Al}_{9} \mathrm{Si}_{2} \mathrm{Fe}_{2}$ in the solute boundary layer just ahead of the primary aluminum phase stimulates nucleation of eutectic silicon and serves to initiate the eutectic reaction. They show further that, for $\mathrm{Fe}$ levels low enough $(\sim 0.0032$ wt.\%) to preclude $\mathrm{Al}_{9} \mathrm{Si}_{2} \mathrm{Fe}_{2}$ nucleation, a refined $\mathrm{Si}$ structure is observed [202], presumably due to the increased undercooling associated with more sluggish nucleation. These assertions have generated some controversy [202205] and, while the role of the $\mathrm{Al}_{9} \mathrm{Si}_{2} \mathrm{Fe}_{2}$ phase (compared to the role of other phases, such as AlP) remains unresolved, trace levels of $\mathrm{Fe}$ appear to have a distinct effect on the eutectic structure.

\subsection{Outlook: pattern selection in coupled growth}

Clearly, the further study of the dynamics of 3D patterns of lamellar and fibrous eutectics is critical to understand the mechanisms leading to spacing selection. In particular, the role of topological defects (lamella terminations, lamellar fault lines, grain boundaries) and the effects of external forces (convection, lateral temperature gradients), crystalline anisotropy and thermal stresses developing at the $\alpha \beta$ interfaces need to be further clarified. The effect of a third component on the stability of eutectics needs to be investigated in off-eutectic compositions in which one of the phases is a compound, and a theoretical model that includes contributions of interface energy and interface kinetics for the compound phase needs to be developed. One open question in this area pertains to the mechanism that sets the eutectic colony size. The stability of eutectic microstructures at high growth rate has also been examined for systems in which the solute-partitioning coefficient is small. This treatment needs to be extended to the case in which non-equilibrium effects are present at the interface.

Microstructure and phase competition (e.g. from eutectic to dendrites, from eutectic to glass) are other subjects of great practical and scientific interest. For multi-phase eutectic coupled growth, a classification of the observed microstructures needs to be established and microstructure selection maps need to be developed. For irregular eutectics, a better understanding of the 3D morphology and associated chemical and thermal fields is required. In addi- 
tion, further investigation into the faceted phase growth mechanisms in $\mathrm{f}-\mathrm{nf}$ (irregular) eutectics and the role of crystal defects, such as twin boundaries, is needed. The role of anisotropy on the maximum stable spacing needs to be established theoretically and experimentally.

Since the selection of peritectic structures strongly depends on the dynamics of nucleation and growth, theoretical models are required to precisely predict the selection of microstructures. These studies, however, have been undertaken in systems in which some convection effects were present. Experimental studies under purely diffusive growth conditions are required to unambiguously validate the model that is based on diffusive growth only.

Progress toward these goals can be made by combining new experimental and simulation techniques. In particular, phase-field modeling is expected to be instrumental in connecting interfacial mechanisms with local 3D microstructural evolution. However, progress on phase-field treatments of $\mathrm{f}-\mathrm{nf}$ interfaces is needed: such models are available for single-phase solidification only [206-208], and have remained restricted to linear interface kinetics. Atomistic simulations such as those described in Section 2 are certainly needed to obtain quantitative information about interface kinetics of faceted interfaces, such that ultimately faceted interfaces with multiple growth mechanisms and nonlinear kinetics can be incorporated into phase-field models of $\mathrm{f}-\mathrm{nf}$ eutectic growth.

These fundamental issues should be addressed if our understanding of chemical modification, mechanical vibrations and applied electric or magnetic fields are to be effectively utilized to develop new alloys and/or growth morphologies offering enhanced properties. For example, it would be highly interesting to find a way for the production of a truly spherulitic Si morphology in Al-Si castings, in analogy with ductile (nodular) cast iron. In this context, a paper on detailed phase-field modeling of spherulites is relevant [210].

Finally, an interesting topic for further studies is the relation between eutectic growth (mostly of $\mathrm{f}-\mathrm{nf}$ type) and metallic glass formation. When the partition coefficients of both eutectic phases are very small, when the eutectic temperature is low and the glass transition temperature is high, a eutectic-to-glass transition is often observed at high solidification rates. While considerable effort has been directed toward understanding and predicting glassforming ability in metallic alloys [209], this topic has not generally been treated within the context of growth. Clearly, a better understanding of nucleation and growth of eutectics competing with glass formation would be helpful in the search for optimal glass-forming alloys.

\section{Microstructure and convection}

The work presented in the earlier sections was centered on diffusional phase transformations. In real situations, however, convection is omnipresent and often plays a dominant role in solidification microstructure for- mation. The last decade has seen a flurry of activities in the area of convection-microstructure interaction. The research has benefited not only from novel experiments, but even more so from the recently emerged capability to perform direct numerical simulations of solidification with convection on a microstructural scale. The following subsections provide an overview of recent research on convection effects on various solidification morphologies, such as plane front, dendrites, eutectics, peritectics and monotectics.

\subsection{Coupled morphological and convective instabilities}

Fluid flow along a solid-liquid interface induces morphological instabilities. Such instabilities have been analyzed theoretically for the case of directional solidification [211-213]. It has been found that the solidification front exhibits slow, spatio-temporal dynamics when the wavelength of the cellular convection is far longer than that of the morphological cells. When the flow is strong, the morphological instability becomes spatially localized and confined at inward flow-stagnation regions on the front. Localized microstructures induced by fluid flow in directional solidification were also observed experimentally in both transparent and metallic alloys [214-216]. The realtime observations revealed two types of localized patterns of a generic nature: focus type and outward hexagonal flow type [214]. A delay of morphological instability, due to solutal convection, was observed downstream of well-oriented solid ridges [216].

Coupled convective and morphological instabilities during directional solidification of an alloy have also been simulated using the phase-field method [217-219]. The simulations revealed the importance of lateral solute segregation, resulting from the gravity-induced flow, on the stability of the interface. For the cases slightly above the onset of instability, the morphologies of the shallow cells are affected by the convection as well. Cells with different wavelengths and depths coexist due to flow-induced segregation. The unique feature of these phase-field simulations is that they show the full coupling of long (convective) and short (morphological) wavelength instability modes beyond the linear stability limit. The planar to cellular transition during upward directional solidification was investigated experimentally using a transparent succinonitrile-acetone alloy [220]. Buoyancy-driven melt convection can significantly stabilize the planar morphology. At the plane-to-cell transition, quasi-hexagonal and elongated cells exist simultaneously, both at grain boundaries as well as inside the grains. With increasing solidification length, a quasi-hexagonal pattern of randomly rearranging cells covers the interface. The cell size varies over a wide range and no wavelength selection was found. These and other complexities still do not allow for a complete and unified understanding of coupled morphological and convective instabilities. From a numerical simulation point of view, the simultaneous presence of short and long wavelength 
instabilities represents a major challenge still to be conquered.

\subsection{Convection effects on dendritic growth}

\subsubsection{Dendrite tip growth}

While tip kinetics during free dendritic growth into a quiescent undercooled melt are reasonably well understood, the elucidation of the well-documented effects of melt convection $[221,222]$ has remained an important challenge. Phase-field models have been developed [223-225] and widely used to simulate solidification in the presence of fluid flow [226-236]. Other methods, such as the sharp-interface tracking technique [237-239], lattice Boltzmann models [240-243], cellular automaton techniques [244-246] and Monte Carlo simulations [247], have also become available.

Quantitative phase-field simulations of free dendritic growth in a pure undercooled melt have been carried out in three dimensions [229,233]. Fig. 11 shows a simulated 3D morphology at the limit of current computational capabilities. These studies have shown that fluid flow modifies the convective heat transport away from the dendrite tip in good agreement with an analytical solution [233,248] that replaces the Ivantsov diffusive transport relation mentioned in Section 3; modifications of the Ivantsov transport theory have also been developed based on boundary layer or stagnant film type models of the effects of natural convection on the heat and solute transport at the dendrite tip [249,250]. For the range of flow velocities and melt undercoolings investigated, and in the limit of vanishing interface kinetic effects, the dendrite tip selection parameter $\sigma$ was found to be unaffected by the flow. This finding is consistent with solvability theory [248], which predicts that $\sigma$ is only altered when the flow velocity is much larger than the dendrite growth rate. Additional advances in numerical techniques are needed to explore higher flow velocities where $\sigma$ is predicted to deviate from the diffusion value

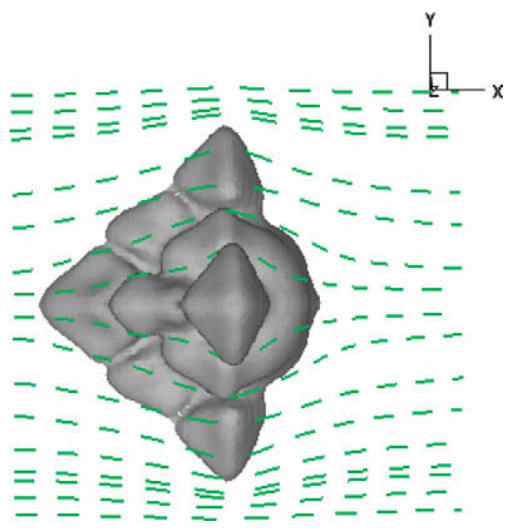

Fig. 11. Three-dimensional phase-field simulation of free dendritic growth of a pure substance into an undercooled melt flowing around the crystal (from left to right); in the simulation, the crystal was held fixed in order to study the effect of the flow relative to the crystal (figure courtesy of C. Beckermann, University of Iowa [233]).
[248]. The effects of convection on dendritic growth in the high tip velocity regime and on dendrite orientation selection, as reviewed in Section 3, also still need to be investigated in more detail.

Dendrite growth in a convective environment during directional solidification of binary alloys has been modeled phenomenologically by taking into account the local growth conditions ( $V, G$ and local composition) and an effective diffusion coefficient that depends on the confinement (e.g. specimen diameter) [124,251]. The stability parameter $\sigma$ was found to be the same for solidification with or without convection only when the effective diffusion coefficient was used to compute $\sigma$ in the case with convection. It is possible that the dendrite tip operating state is influenced by the overlap of diffusion fields from nearby dendrites in directional growth, and thus is quantitatively different from that for free growth. However, this difference, if present, has not been systematically investigated.

Dendrite tip growth during settling of equiaxed grains in an undercooled melt was investigated in several experimental studies involving transparent alloys [252-254]. Measurements were performed to investigate the effect of the flow (or settling) velocity and of the angle of the flow with respect to the dendrite growth direction [254]. While the dendrite tips pointing into the flow generally grow at a velocity that is greater than the value predicted by the standard diffusion theory, the dendrite tips in the wake of the crystals grow at a velocity that is substantially less than in the absence of flow. When the flow is at an angle normal to the dendrite axis $\left(90^{\circ}\right)$, the tip growth velocity is unaffected by the flow. Interestingly, the average growth velocity of all six primary dendrite arms of a settling equiaxed crystal was found to be in almost perfect agreement with the value predicted by the standard diffusion theory, despite the presence of considerable settling speed changes and crystal rotation.

\subsubsection{Dendrite arm development and spacings}

The effect of convection on the columnar (primary) dendrite trunk or cell spacing in directional solidification has also emerged as a subject of strong research interest. Numerous experimental studies have been performed where the flow is induced by thermal and/or solutal buoyancy forces [255-263]. While these experimental investigations reveal several interesting phenomena, few general statements regarding the convection effect can be made. The primary spacing either increased or decreased compared to purely diffusive conditions (as in a zero gravity environment). This is related to the complex nature of the natural convection: in some alloys, the rejected solute causes an increase in the melt density, while in others it causes a decrease. This results in different microscopic convection patterns between the dendrite arms near the tips. The direction of the gravity vector relative to the solidification direction and the existence of lateral thermal gradients also play important roles. Macroscopic convection on the scale of the crucible induces long-range variations in the 
solute concentration that affect the primary spacing as well. Often, convection is unsteady or even oscillatory, and steady-state growth conditions and stable arm spacings are never achieved.

The complex nature of the effects of thermo-solutal natural convection on the primary dendrite (trunk) spacing is well illustrated by the phase-field simulations of Steinbach and co-workers $[264,265]$ for directional solidification of an Al $-4 \% \mathrm{Cu}$ alloy. As shown in Fig. 12(a), when the dendrite array is solidified upward, segregated $\mathrm{Cu}$-rich melt of a higher density flows downward along the dendrite trunks and accumulates in the space between the primary arms. This solute accumulation by the flow causes an increase in the primary arm spacing to $600 \mu \mathrm{m}$, compared to $330 \mu \mathrm{m}$ under purely diffusive conditions. The flow is confined to the interdendritic region and is relatively weak, since the thermal gradient ahead of the growth front induces stable density stratification. On the other hand, during downward solidification (Fig. 12(b)), strong and highly unsteady convection exists in front of the growth front, since this configuration is both thermally and solutally unstable. This flow washes away the rejected solute between the dendrite trunks and causes a decrease in the primary spacing to $270 \mu \mathrm{m}$. Other phase-field simulation studies have investigated the inclination of dendrite arms
$[235,266,267]$ and the selection of columnar grains in the presence of a shear flow [236]. While these numerical studies represent key contributions to the understanding of convection effects in directional solidification, additional work remains to be performed. In particular, these studies need to be extended to three dimensions and more realistic growth conditions.

Extensive experimental studies on the effect of convection on the dendrite morphology during directional solidification of alloys have been performed by using magnetic fields to induce a flow of known strength [268-271]. As shown in Fig. 13(a), the application of magnetic fields of different strengths allowed for the validation of a relation $[272,273]$ that predicts the primary dendrite arm spacing as a function of the flow velocity. Here, the flow induced by the rotating magnetic field causes the primary dendrite arm spacing to decrease. In addition, these experiments confirmed previous phase-field simulations [274] of the effect of convection on the secondary dendrite arm spacing. The interdendritic flow induced by the magnetic field increases the secondary spacing, and the regular coarsening exponent of $1 / 3$ in the absence of flow increases to $1 / 2$ (Fig. 13(b)). More complex microstructural effects are present at very high magnetic field strengths (up to $10 \mathrm{~T}$ ) $[275,276]$. Depending on the growth rate, the magnetic field a

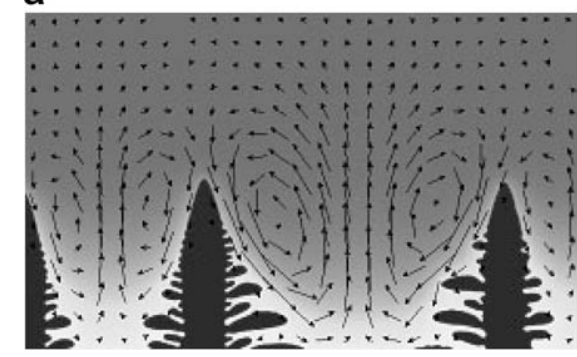

b

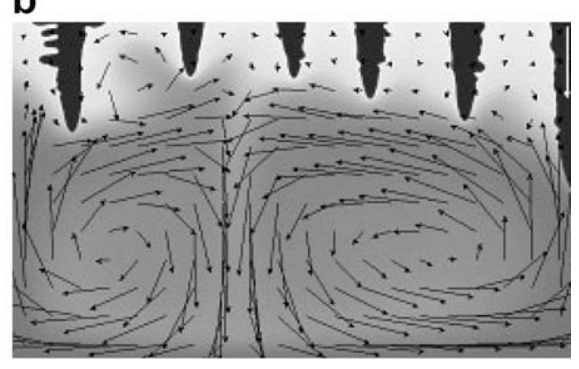

Fig. 12. Two-dimensional phase-field simulations of the effect of thermo-solutal convection on the primary dendrite arm spacing in an Al-4\% $\mathrm{Cu}$ alloy directionally solidifying with a thermal gradient of $10 \mathrm{~K} \mathrm{~mm}^{-1}$ and a velocity of $40 \mathrm{~mm} \mathrm{~s}^{-1}$; (a) upward solidification and (b) downward solidification. The grey shades indicate that the solute concentration in the melt varied between $4 \% \mathrm{Cu}$ (dark) and $7 \% \mathrm{Cu}$ (light) (figure courtesy of I. Steinbach, ACCESS, Aachen [265]).
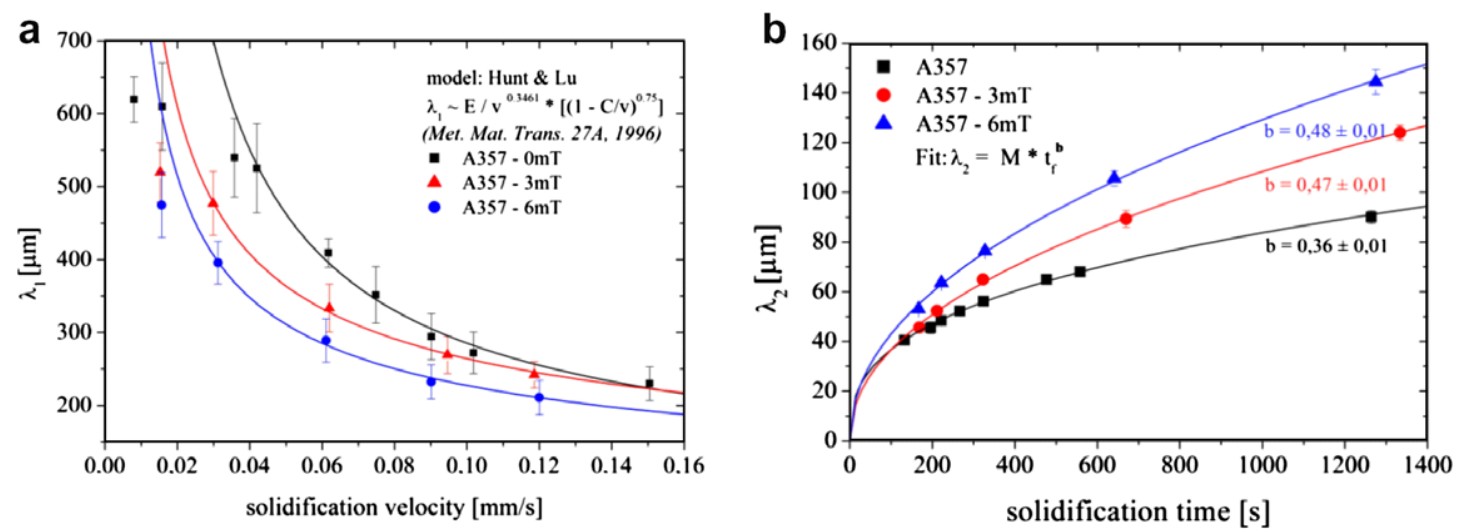

Fig. 13. Effect of a rotating magnetic field on (a) the primary and (b) the secondary dendrite arm spacings in a directionally solidified A357 aluminum alloy (figure courtesy of L. Ratke and S. Steinbach, DLR, Cologne [270,271]). 
causes tilting, rotation and general disorder in the dendrite pattern, an increase in the primary dendrite arm spacing along with an enhanced side-branch structure and even a transition to an equiaxed grain structure. These phenomena were attributed to the thermo-electromagnetic convection caused by the high magnetic field. Overall, the use of magnetic fields in experiments designed to understand the role of convection during solidification has resulted in significant progress during the past 10 years, and additional experimental work along these lines would be highly desirable.

In addition to the arm spacings during directional solidification, gravity-driven convection can also influence the side-branch characteristics of freely grown equiaxed dendrites, and significant differences to corresponding microgravity experiments have been observed [277-279]. Forced convection, as in DC casting of aluminum alloys, can affect the formation of feathery grains made of twinned dendrites [280]. Finally, the dendritic microstructure can also be altered by convection that is induced by vibrations, and dendrite fragmentation can result [260,281].

\subsubsection{Columnar-to-equiaxed transition}

Direct numerical simulations of the columnar-to-equiaxed transition (CET), using the phase-field method, are now possible for purely diffusive conditions and in two dimensions [282]. However, corresponding numerical simulations taking into account the presence of melt flow, dendrite fragmentation and movement of small equiaxed grains are still awaiting the availability of increased computational capabilities. Experimental investigations of dendrite fragmentation in metallic alloys solidifying under natural (non-forced) convection conditions have recently been performed using synchrotron radiography $[283,284]$. This very powerful experimental technique allows for real-time, direct observation of solidification and convective transport in metals on a microstructural length scale. It was found that fragmentation of higher-order dendrite branches could result from solute-rich liquid flowing from the tips into open regions of the dendritic network [284]. The effect of the transport and settling of fragments on CET was observed in detail [283].

In industry, dendrite fragmentation is often accomplished by electromagnetic stirring of the melt, and considerable grain refinement can be achieved. For such an application, a dendrite fragmentation criterion has been developed [285]. However, the fragmentation of dendrites in the presence of flow certainly deserves much additional research attention. Also, the subsequent evolution of the fragments under convective conditions [286] has not been studied sufficiently.

\subsection{Convection effects on multi-phase solidification}

\subsubsection{Eutectic growth}

Several models have been developed for the lamellar spacing during eutectic growth in the presence of melt flow
[287-289]. They generally predict an increase in the lamellar spacing with convection intensity. However, the effect of buoyancy-driven convection, or its absence in microgravity, on eutectic microstructure has largely remained unexplained [290]. Thus, it was hypothesized that freezing rate fluctuations due to irregular convection might be responsible for spacing adjustments in a fibrous microstructure [290]. An increase in freezing rate must cause new fibers to form, either by branching or by nucleation, and a decrease in freezing rate would cause fibers to terminate by overgrowth of the matrix phase. However, this mechanism was found to result in a finer microstructure, instead of the increase in the spacing that is generally observed with increasing convection. Thus, a different mechanism was proposed whereby the freezing rate oscillations cause the average interfacial melt composition to deviate from the eutectic because the system does not solidify at the extremum. This results in the formation of a composition boundary layer of sufficient thickness that the system would become sufficiently sensitive to convection [290]. Direct numerical simulation of eutectic solidification with melt convection should become possible in the near future and could contribute much to the understanding of the above and other effects.

The effect of gravity-induced fluid flow on eutectic microstructure has also been investigated experimentally for Al- $\mathrm{Cu}$ alloys [291]. Different convection modes were achieved by varying the composition from hypo- to hypereutectic. Single-phase-to-eutectic, lamellar-to-rod-eutectic and rod-to-lamellar-eutectic transitions were observed. The fluid flow effect on eutectic spacing in eutectic or near-eutectic alloys was found to be very small, whereas it increases the eutectic spacing in hypoeutectic alloys due to the increase in the effective diffusion coefficient value. The spacing can increase or decrease in hypereutectic alloys since the bulk composition changes continuously due to the rejection of lighter $\mathrm{Al}$, so that the spacing value depends on the solidification fraction. Models were developed to predict the variations in eutectic microstructure caused by fluid flow.

Density-change-driven flow was found to have a potentially strong effect on eutectic growth [292]. When the densities of the two solid phases are different, there are flow components both perpendicular and transverse to the solid-liquid interface. The direction of the transverse flow is always from the less dense solid phase to the denser solid phase. Assuming equal densities of the two solid phases can lead to large errors in the phase volume fractions. For some alloys, the minimum undercooling and the lamellar spacing differ substantially from the values given by the JacksonHunt theory for equal densities. For $\mathrm{Fe}-\mathrm{C}$ alloys, where the $\beta$ phase has a lower density than the $\alpha$ phase, the lamellar spacing decreases by a factor of 1.5 due to densitychange-driven flow. For most other alloys, the density of the $\beta$ phase is higher than that of the $\alpha$ phase and the lamellar spacing increases, as one would expect in the presence of flow. 


\subsubsection{Peritectic and monotectic growth}

Convection effects in peritectic systems have continued to attract research interest [182,293-295]. Convection was found to reduce the spacing in a banded peritectic microstructure. A variety of novel pattern-forming processes due to the coupling of convection and phase change were discovered. The coupling of flow oscillations near the interface with solidification results in ordered layered structures. Strong oscillatory convection produces a large treelike primary phase that is surrounded by the peritectic phase matrix. Nucleation and convection effects in peritectic systems have also been examined in recent phase-field simulations [296].

The important role of convection during monotectic growth has been known for some time. Phase-field simulations of monotectic solidification with convection in both liquid phases were performed to better understand the full complexities of the transport processes involved [297]. For fibrous monotectic growth, a new relation for the fiber spacing in the presence of Marangoni convection was derived $[298,299]$. A static magnetic field was used to eliminate convection during directional solidification of an AlBi monotectic alloy, and the fiber spacing was observed to decrease [300].

\subsection{Outlook: microstructure and convection}

One of the main present challenges is to develop a quantitative understanding of convection effects that strongly influence dendrites in actual castings. While significant progress has been made during the last decade in unraveling the complex effects of convection on solidification microstructure development, much of the understanding remains incomplete or fragmented. Most of the existing theories or experimental findings are only valid in very specific situations or narrow regimes. For example, it is still not clear how to calculate the primary and secondary dendrite arm spacings in a mushy zone for any value and direction of the local flow velocity. This is important not only for predicting the microstructure in solidified materials, but also for determining the permeability of the mushy zone during solidification and for modeling phenomena such as macrosegregation, porosity formation and hot tearing. Experiments and numerical simulations where the flow is externally imposed and well defined promise to yield the most insight, and additional progress in both experimental (e.g. using magnetic fields for flow control or by in situ observations with synchrotron radiation) and numerical techniques can be expected. However, in most solidification processes convection is caused by solidification itself and the flow is heavily influenced by the developing microstructure. Furthermore, convection is usually unsteady and of a long-range nature, and can thus change the environment in which the microstructure grows.

Studies of the possibilities for improving processing by modifying the interaction between flow fields and interface morphologies are scarce. For example, there is a strong need for a more quantitative model of equiaxed growth in a constitutionally undercooled boundary layer of a columnar dendritic growth front that includes dendrite fragmentation, transport, dissolution and growth of fragments in the presence of fluid flow.

The limited range of processing parameters for which aligned monotectic composites can be grown is controlled by fluid flow, but the precise mechanisms remain to be determined. There has been progress in treating the effect of simple flows on linear morphological stability, but extension into the nonlinear regime remains to be done. Three-dimensional time-dependent simulations of fluid flow are now possible, but become difficult for complex flows that exhibit flow transitions. These confounding issues need to be considered more carefully to make any real progress.

\section{Microstructures and defects at high solid fractions}

The kinetics of dendrite tips and the dynamics of eutectic fronts have been the focus of much of the attention of physicists and materials scientists (see Sections 3 and 4). Although these are important topics for the understanding and modeling of solidification microstructures, phenomena occurring deep in the mushy zones are just as important in the formation of two major solidification defects, namely microporosity and hot tearing. This is particularly the case for dilute alloys with a large solidification interval, $\Delta T_{0}$. In such alloys, the fraction of solid $g_{\mathrm{s}}$ at which a non-equilibrium eutectic might form from the last liquid is close to unity. Solidification shrinkage associated with the formation of this eutectic might not be compensated by liquid flow, thus leading to the formation of shrinkage porosity. The space available for the growth of such pores is dictated by the morphology of the primary phase, and thus coarsening is an important parameter. Further, when $g_{\mathrm{s}}$ is typically larger than 0.9 , the primary phase undergoes a morphological change from isolated solid elements (dendrites or grains) surrounded by continuous liquid films, to a continuous solid network with isolated liquid droplets. This coalescence phenomenon leads to the percolation of the solid, i.e. to the formation of a cluster of grains of increasing size, finally equal to that of the considered domain. This transition is very critical in the formation of hot tears or hot cracks.

This section briefly reviews recent progress made in the characterization and understanding of microstructure evolution at high solid fractions and of formation of microporosity and hot tearing.

\subsection{Microstructure evolution at high solid fractions}

On the experimental side, serial sectioning [301-303], Xray tomography [304-306] and in situ X-ray radiography $[284,307,308]$ have emerged over the past 10 years as powerful techniques for the observation of microstructure evolution in metallic alloys at low and high solid fractions. 
While the first technique can be applied to solidified specimens only, the advent of high-intensity synchrotron X-ray sources has allowed in situ observations of microstructure formation in metallic systems, mainly low-absorbing elements such as aluminum. Using serial sectioning, Voorhees and co-workers [309] observed the evolution of microstructures, in particular the particle size distribution and the number of particle contacts during isothermal holding of $\mathrm{Sn}-\mathrm{Pb}$ alloys. They compared their results with phase-field predictions [310]. Similar observations have been made by Suéry and co-workers [305] at higher temperatures for Al$\mathrm{Cu}$ alloys, but using an in situ tomography technique. As a main conclusion of these studies, the evolution of the microstructure at a high solid fraction is dictated not only by Ostwald ripening, but also by coalescence (or bridging) of, and growth of the necks in between, individual particles. Besides the observation of individual coarsening or coalescence events [311], new statistical approaches are required for the representation of the solid-liquid ( $\mathrm{s} \ell$ ) interface evolution. For example, after determining the mean curvature $H$ and the Gaussian curvature $K$ at each point of the solid-liquid interface, Voorhees and co-workers [312] use a $H-K$ space representation to follow the evolution of the surface density of these points.

While coarsening phenomena have been investigated fairly thoroughly from an experimental and a theoretical point of view [312], coalescence or bridging of two particles or dendrite arms has not been studied to any great extent. Being the opposite of grain boundary wetting, this last phenomenon is primarily dictated by the Gibbs-Smith condition, which compares the grain boundary energy $\gamma_{\mathrm{gb}}$ and twice the solid-liquid interfacial energy $2 \gamma_{\mathrm{s} \ell}$ [313]. Based on simple thermodynamic considerations [314], it can be shown that, for $\gamma_{\mathrm{gb}}<2 \gamma_{\mathrm{s} \ell}$, a grain boundary is "attractive", i.e. solid bridges are established as soon as the two solidliquid interfaces of the grains get within an interaction distance of the order of the thickness $\delta$ of the diffuse solidliquid interface. However, when $\gamma_{\mathrm{gb}}>2 \gamma_{\mathrm{s} \ell}$, the grain boundary is "repulsive" and bridging requires an additional coalescence undercooling, $\Delta T_{\mathrm{b}}=\left(\gamma_{\mathrm{gb}}-2 \gamma_{\mathrm{s} \ell}\right)$. $\left(\Delta S_{\mathrm{f}} \delta\right)^{-1}$, where $\Delta S_{\mathrm{f}}$ is the volumetric entropy of fusion. For an alloy, it has been shown that liquid films remain at a grain boundary until a coalescence line (or surface), parallel to the liquidus but located $\Delta T_{\mathrm{b}}$ below, is reached by a combination of cooling and back-diffusion. This approach was substantiated by phase-field simulations [314] and more recently by MD calculations [315].

Coalescence in alloys can be modeled in detail only for very small volume elements since it involves three widely different length scales, associated with diffusion, capillarity and (real) thickness of the diffuse interface. However, its influence on the gradual formation of a coherent solid manifests itself on the scale of a fairly large population of grains since it also includes statistical aspects related to the crystallographic orientation and the respective position of the grains. Therefore, in order to approach such problems, granular or discrete element methods (DEM) have been developed recently [316-318]. In such models, grains assumed to be globular are randomly distributed in space with random crystallographic orientations. A simplified 1D microsegregation-coalescence model can then be used to predict the solidification of each individual grain and of the liquid channels in between them. As clusters of grains of increasing size form, the gradual transition from continuous liquid films to a continuous solid network can be modeled (Fig. 14).

\subsection{Mechanical properties of the mushy zone}

As clustering occurs during solidification, the rheology of the mushy zone gradually evolves from that of a viscous fluid with grains in suspension at low $g_{\mathrm{s}}$, to that of a fully solid material near $g_{\mathrm{s}}=1$. At intermediate values of $g_{\mathrm{s}}$ when liquid films are still interconnected, the drastically different rheological behaviors of the solid skeleton and of the intergranular liquid make the mushy solid to appear compressible, even though each individual phase is not. For such complex materials, two-phase average mass and momentum equations have been derived [319-321], but they must be complemented by constitutive equations. While that of the liquid can be approximated by Darcy's law, the viscoplastic compressible behavior of the mushy solid is described by a model in which the pressure in the solid appears, in addition to the equivalent von Mises

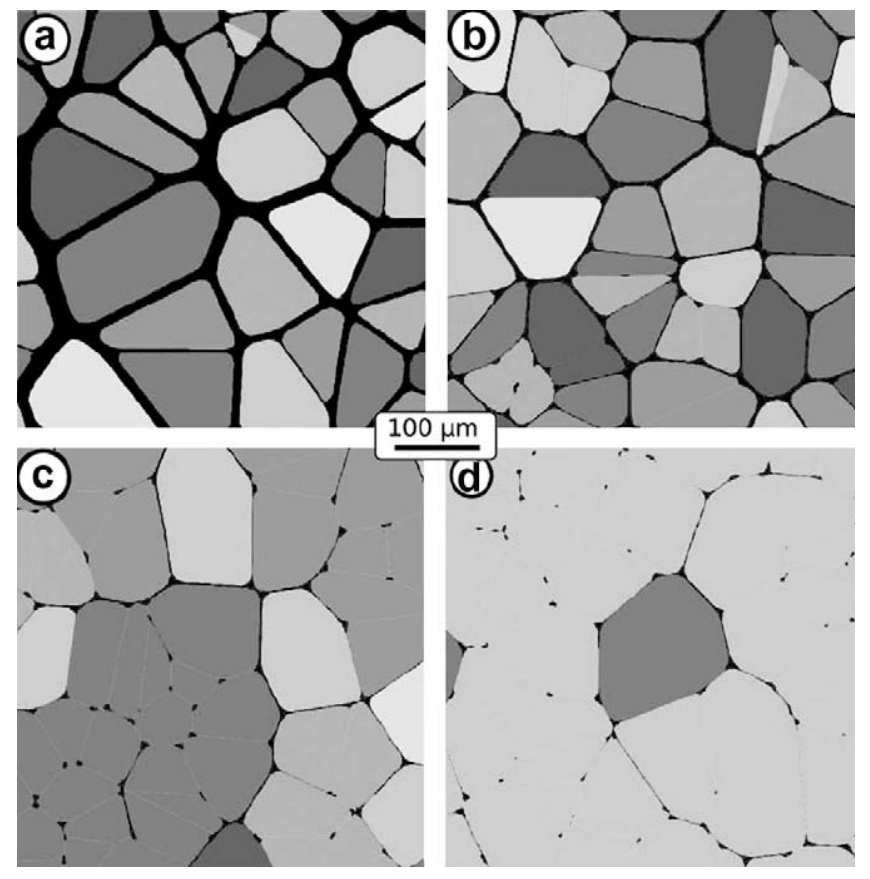

Fig. 14. Computed grain structure evolution in two dimensions in a directionally solidified $\mathrm{Al}-1$ wt.\% $\mathrm{Cu}$ alloy. The four images (a-d) represent various transverse sections of increasing volume fraction of solid of a directionally solidified specimen. They clearly show the gradual percolation, i.e. the formation of increasingly large size clusters of grains. The connectivity of the grains or clusters appear with various grey levels while the liquid is black. (After Vernède et al. [316,317].) 
stress. Further details of the rheological behavior of a mushy solid can be found in Refs. [322-324].

\subsection{Microporosity formation}

Shrinkage microporosity in alloys is a result of a lack of feeding of the mushy zone, i.e. the density increase associated with solidification cannot be fully compensated by an interdendritic fluid flow opposite to the displacement of the isotherms [325]. As this flow induces a pressure drop in the interdendritic liquid when the temperature decreases, the solubility limit of gases such as hydrogen or nitrogen decreases. Simultaneously, their actual composition in the liquid increases due to segregation and might exceed at some point their solubility limit, thus allowing pore nucleation. However, the contributions of gas diffusion and capillarity must be accounted for the nucleation and the growth of pores. Fairly comprehensive microporosity models [326-331] have been developed since the first work of Piwonka and Flemings [332], but they will not be detailed here. However, because it is directly linked with the morphology of the primary phase, the capillarity contribution is briefly discussed here. Again, X-ray tomography has been instrumental to reveal the very complex and interconnected morphology of shrinkage porosity in 3D (see Fig. 15). Two-dimensional sections make them appear as small, disconnected holes. The highest curvature of such pores corresponds to the portion of its surface in contact with the interdendritic liquid, since the gas-liquid (or gas-solid) interfacial energy $\gamma_{\ell g}$ is typically one order of magnitude larger than $\gamma_{\mathrm{s} \ell}$. In $\mathrm{Al}-\mathrm{Cu}$ alloys, radius of curvature as small as $5 \mu \mathrm{m}$ have been measured thus inducing a Laplace overpressure of about $400 \mathrm{kPa}$ with respect to the surrounding liquid. Such contributions must absolutely be accounted for in porosity models if realistic simulations are to be produced.

\subsection{Hot tearing}

If microporosity is the major defect in shape casting, hot tearing is certainly the major defect of continuous or semicontinuous casting processes and of welding [325]. Although similar to shrinkage-induced microporosity since it occurs in large solidification interval alloys, hot tearing involves tensile stresses in the partially coherent solid. Compared to microporosity models, hot tearing was approached with very simple criteria related, for example, to the solidification interval [325] or the feeding ability [333] of the alloy. Clyne and Davies [334] recognized the importance of the microstructure in the formation of this defect, namely the persistence of continuous liquid films at grain boundaries in a region of the mushy zone where feeding is nearly impossible. They defined a criterion based on the time spent by the mushy zone in this critical region of the mushy zone, typically for $0.9<g_{\mathrm{s}}<0.99$. However, when applied to various solidification processes, this criterion fails to predict the right trend $[335,336]$. Other hot

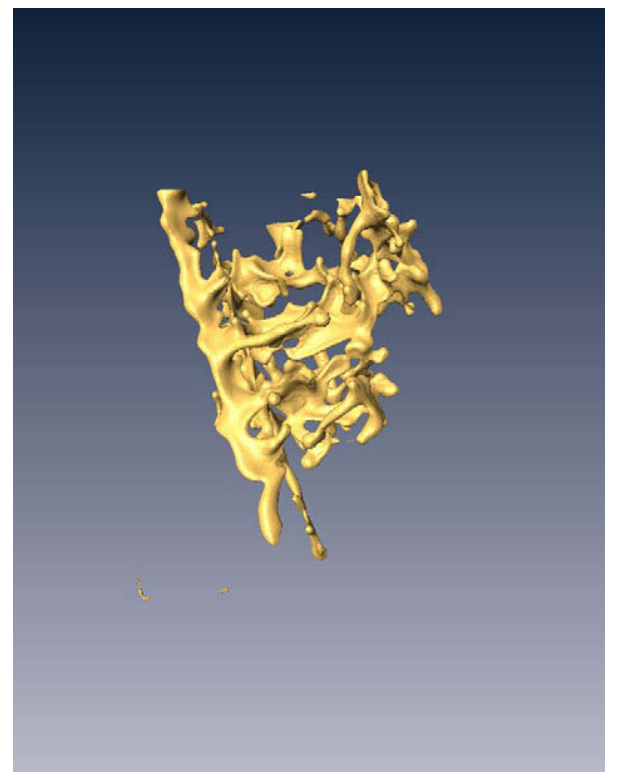

Fig. 15. Three-dimensional high-resolution X-ray tomography of a shrinkage porosity formed in an $\mathrm{Al}-4.5 \mathrm{wt} . \% \mathrm{Cu}$ alloy [403].

tearing criteria based on the stress, strain or strain rate experienced by the mushy zone $[337,338]$, sometimes with a normalization with the cooling rate, have been developed, but the mechanisms of straining and feeding of the mushy zone have been approached only recently using two-phase models.

The first, yet simple, two-phase approach for the prediction of hot tearing was derived in 1999 by Rappaz, Drezet and Gremaud (RDG) [339]. Similar to other criteria for porosity formation [340], this approach considers steadystate directional solidification in a given thermal gradient and a strain rate $\dot{\varepsilon}_{s \perp}$ in the perpendicular direction. The pressure drop $\Delta p_{\ell}$ from the liquidus $T_{\text {liq }}$ to a coherency temperature $T_{\text {coh }}$ can be calculated, $T_{\text {coh }}$ being defined as the temperature at which continuous liquid films disappear. This RDG criterion can also be used under non-steadystate conditions by first calculating the strain rate in the fully solid part and then feeding in the mushy zone considering the strain rate component perpendicular to the thermal gradient [341]. Although this criterion compares fairly well with experiments [336], it has two main weaknesses: (i) the strain rate in the mushy zone is not well defined, as it is taken from the extrapolation of a deformation calculation performed for a fully solid material; and (ii) the model is very sensitive upon $T_{\mathrm{coh}}$.

The first limitation of the RDG criterion was removed using a real two-phase formulation in which the mechanical behavior of the compressible mushy solid is accounted for [324,342] (see Section 6.2). In such models, the calculation of the velocity $\mathbf{v}_{\mathrm{s}}$ of the mushy solid is coupled with that of the pressure drop $\Delta p_{\ell}$ in the liquid, the latter now including the contributions of solidification shrinkage, as for microporosity (see Section 6.3), and of the compression/expansion of the mushy solid. The second limitation 
is strongly related to the evolution of the microstructure at high $g_{\mathrm{s}}$ value.

Because they are based on continuous one- or two-phase media, hot tearing approaches neglect the importance of localization of strains at grain boundaries. Yet the effect of grain boundaries on hot tearing was clearly demonstrated by welding two single crystals of nickel-based superalloy under identical conditions but with an increasing tilt boundary misorientation (Fig. 16) [343]. These observations showed that hot cracks formed only when the misorientation exceeded a given value, which was then interpreted based on a grain boundary energy and coalescence model. Such observations clearly indicate the necessity to account for the microstructure evolution at a high solid fraction, and thus granular or DEM approaches really should be developed. Although limited yet to $2 \mathrm{D}$ geometries, such developments have already been undertaken and proved their usefulness [316-318,344].

\subsection{Outlook: microstructures and defects at high solid fraction}

Over the past 40 years, theories and models have focused a lot on the early stages of solidification (formation of dendrites, eutectics, etc.). Yet the evolution of the microstructure at high solid fraction is a key parameter for the prediction of defects such as microporosity and hot tearing. Besides the improvement of commercial solidification codes for the prediction of such defects, our understanding of microstructure evolution at high solid fraction must be improved. Along this line, a detailed modeling of coalescence based on molecular dynamics or phase-field crystal methods, correlated with in situ X-ray tomography observations, is necessary. For alloys, it might be necessary to couple such methods with the phase-field technique if one desires to model simultaneously diffusion in the solid phase and preferential solute segregation at grain boundaries. Based on such micromodels, the percolation of an ensemble of grains will have to be studied in three dimensions in order to capture the gradual transition of the mushy zone under more realistic conditions. For example, in two dimensions, either the solid or the liquid phase is continuous, whereas in three dimensions both can be continuous at the same time. This will require the development of dedicated 3D granular or discrete element models that encompass solidification and coalescence. We have almost all the tools necessary for the study and modeling of the growth of microporosity in a well-developed dendritic network. Fast X-ray tomography combined with multi-phasefield simulations will definitely give a quantitative assessment of the important contribution of capillarity during pore formation. Hot tearing modeling will also go along the same direction of granular or discrete element models. Such methods have already been employed successfully for a whole range of problems (e.g. sintering, fragmentation, soil mechanics, granular flows). A better knowledge of the mechanical properties of the semi-solid material and the characterization of the microstructure near a solid fraction of unity are necessary. A 3D model that encompasses solidification, coalescence, feeding and deformation in the mushy zone has to be developed.

\section{Solid-state transformation parallels}

In the 1950s and 1960s, the distinction between the fields of solidification and solid-solid transformations was not as clearly defined as it is today; for example, back then the same theories were used to study both the growth
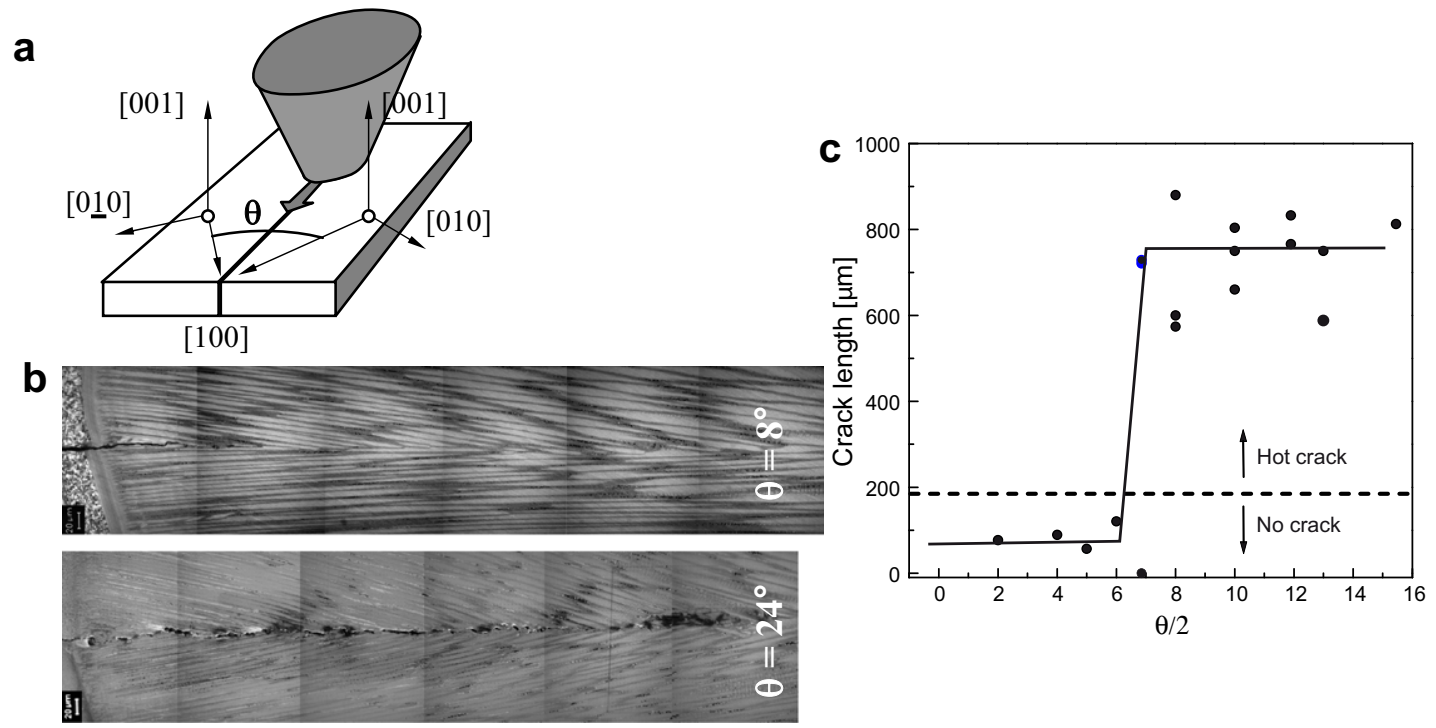

Fig. 16. Hot tearing weld experiment. (a) Experimental setup with laser beam (in grey) used for welding of two nickel-base superalloy single crystals with symmetric orientation with respect to the weld plane (angle $\alpha$ between [100] and weld direction, 2 $\alpha=\theta$; (b) laser welds of two bi-crystals exhibiting tilt grain boundary angles $\theta$ of 8 (above) and $24^{\circ}$. (c) Crack length as a function of grain boundary half angle $\alpha$. Under identical welding conditions, only low angle grain boundary welds with $\theta \leqslant 12^{\circ}$ did not exhibit hot cracking [343]. 
of dendrites and Widmanstaetten plates. In the following years, the fields of solidification and solid-state transformations have deepened and diverged. In recent years, however, there have been a few tentative attempts to close this gap.

The theory of solid-state phase transformations really started with the landmark paper by Zener some 60 years ago [345], which laid the foundation of more quantitative models by Hillert [346], Trivedi [347] and Jackson and Hunt [156]. These models have since been used extensively in solidification research. This section is not a review of solid-state transformations but an attempt to highlight links between the two fields, stressing that there can be fruitful cross-fertilization of ideas and techniques between solidification and solid-state transformation.

There are certain similarities in the evolution of both fields, in that solute transport, interfacial energy and interfacial kinetics [348] have been considered significant in both cases. Similar phenomena include the growth of needles and plates (e.g. dendrites vs. Widmanstaetten structures), coupled growth of eutectics and eutectoids, and massive transformations. There are, however, several major factors that are different: convection effects are crucial in solidification only, whereas strain energy effects often play an important role in solid-state precipitation. In addition, and related to the fact that at least two crystals must meet at the solid-solid transformation front, anisotropies of interfacial free energy and interfacial mobility are generally more pronounced in solid-solid transformations: interfaces are often faceted, requiring the formation and lateral propagation of ledges for their migration [349]. Consequently, the strongest parallels between solidification and solid-solid transformations will be found in faceted growth (for both) on the one hand, and in non-faceted growth (e.g. comparing the motion of "incoherent" solid-state interfaces with that of typical metallic solidliquid interfaces) on the other.

Phase-field modeling techniques that have proved so valuable in solidification science have also been applied to solid-state transformations. The review on the subject by Chen [350] is helpful for work before 2002. Multiscale models are highly promising approaches with the potential to yield quantitative insight into microstructure evolution [109]. A more tractable technique, which can cope with orders of magnitude larger space and time frames, has been developed recently by Elder et al. [351] and is called the phase-field crystal (PFC) method (a review of this method can be found in Ref. [152]).

Most of the experimental studies in solid-solid transformations are carried out isothermally, similar to the solidification of alloys in an undercooled melt. In contrast, directional transformation is used widely in solidification, but has not been very common in the study of solid-state transformations, despite its potential.

In the following the subject is therefore subdivided into directional and isothermal transformations of single- or two-phase alloys. As the topic has not been subject to a recent review, the work presented is not limited to the last decade.

\subsection{Directional transformation}

\subsubsection{Single-phase growth}

Directional transformation (Bridgman type growth) is characterized by an imposed growth rate in a positive temperature gradient. The conditions are chosen such as to avoid nucleation of the product phase in the parent phase and to obtain a macroscopically flat transformation front. This technique allows direct examination of the interface response, i.e. the development of different interface morphologies of an alloy (plane front, cells, dendrites) and related interface/tip temperatures as a function of velocity in a selected temperature gradient [352,353]. Directional transformation of the alloy can be carried out from liquid to room temperature so that the consecutive liquid-solid and solid-solid transformation fronts in a given sample can be studied under well-defined steady-state conditions [354]. In the high-temperature solid-state transformation from $\delta$-ferrite to $\gamma$-austenite in $\mathrm{Fe}-\mathrm{Ni}$ or $\mathrm{Fe}-\mathrm{Cr}$ alloys it has been shown that solidification models apply well to the observed solid-state transformation structures $[354,355]$ as the interface seems to be mobile and the parent $\delta$ has higher diffusion coefficients than $\gamma$. Further, it could be unambiguously shown that in a $\mathrm{Fe}-17.5$ at.\% Co alloy [357] absolute stability [356] of the solid-solid transformation front was reached. In Fig. 17 the growth fronts for two different velocities ( 1 and $10 \mu \mathrm{m} \mathrm{s}^{-1}$ ) can be seen: The $\mathrm{L} / \delta$ fronts (top) of both quenched specimens correspond to plane front growth as they form below constitutional undercooling for solidification. The $\delta / \gamma$ solid-state transformation front (centre) is cellular at $V=1 \mu \mathrm{m} \mathrm{s}^{-1}\left(<V_{\mathrm{a}}\right)$ and planar at $10 \mu \mathrm{m} \mathrm{s}^{-1}\left(>V_{\mathrm{a}}\right)$ [357]. The calculated limit of
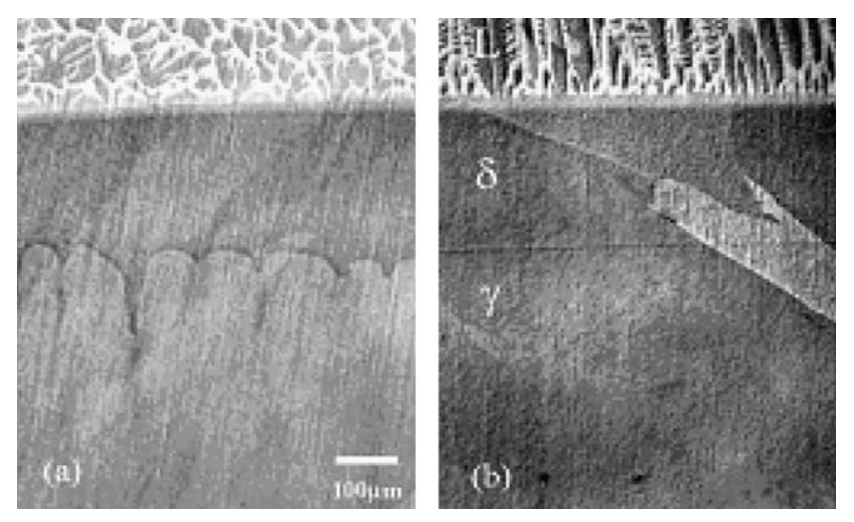

Fig. 17. Consecutive phase changes from top to bottom $(\mathrm{L} / \delta$ and $\delta / \gamma)$ in directionally transformed hypoperitectic $\mathrm{Fe}-17.5$ at.\% Co alloy; $G=18 \mathrm{~K} \mathrm{~mm}^{-1}$. (a, top) Plane front $\delta$ ferrite growing at a smaller rate than the critical velocity for constitutional undercooling for solidification and (a, bottom) cellular $\gamma$ transformed in solid state at $V=1 \mu \mathrm{m} \mathrm{s}^{-1}$. (b, top) Plane front $\delta$ below the critical rate of constitutional undercooling and (b, bottom) plane front $\gamma$ above absolute stability for solid-state transformation at $V=10 \mu \mathrm{m} \mathrm{s}^{-1}$ [357]. In (b) the low temperature austenite-ferrite transformation produced a ferrite grain (light grey). 
absolute stability, $V_{\mathrm{a}}$, for this solid-state transformation is $6 \mu \mathrm{m} \mathrm{s}^{-1}$, in agreement with experiment. One has in Fig. 17(b) the interesting case of two planar transformation fronts: the liquid/ $\delta$ front, which grows at a speed below the limit of constitutional undercooling, and the $\delta / \gamma$ front, which grows above the limit of absolute morphological stability. The reason for this behavior is the huge difference in the diffusion coefficients between the solid and liquid phases. The solid-state plane front grows close to local equilibrium and in steady state the product phase is completely supersaturated as a result of a narrow concentration pile up in ferrite. Such a transformation where the product phase has the same composition as the parent phase is called a "massive transformation". In this sense, two consecutive massive transformation interfaces are observed (Fig. 17(b)). The simultaneous observation of two or more interfaces would provide critical information when strain energy, interface kinetics or rapid solidification effects due to the larger Peclet number in the solid influence the solid-solid interface structure.

\subsubsection{Two-phase growth}

For every three-phase reaction involving one liquid and two solid phases, there exists a solid-state analogue, e.g. eutectic/eutectoid; peritectic/peritectoid; monotectic/ monotectoid. The eutectoid (pearlite) reaction in carbon steels has attracted perhaps the most attention, as these steels are widely used. Eutectoid growth is also amendable to study by directional transformation techniques [358362]; the analogy with eutectic growth is nearly complete.

The models of eutectic growth at low rates have been experimentally verified for both the lamellar [159] and rod eutectic [363] in systems whose required parameters have been accurately measured. For solidification, the scaling laws between velocity and interphase spacing and between undercooling and velocity were found to follow the model based on diffusion in the parent phase including capillarity, which are $\lambda^{2} V=$ const. and $\Delta T^{2} \propto V[346,156]$.

In contrast, in eutectoid systems the above equations often agree less well with the experimental data. For pearlite growth, Pearson and Verhoeven [358,359] showed that the scaling laws are obeyed, but that the values of the constants disagree. This discrepancy has been recently re-evaluated by Steinbach and Apel [364] with phase-field calculations to fit experimental undercoolings of pearlite with theory (Fig. 18). Further experiments are necessary to confirm the assumptions of this model.

Carpay [362] has carried out directional transformations in several eutectoid systems, and significant deviations in the exponent in the scaling laws have been obtained. This can be attributed to the various physical phenomena that become important in solid-solid transformation, which include interface diffusion, strain energy, interface kinetics or diffusion in the two new phases. In fact, different models that include these effects have been developed and compared with the experimental results to establish the mechanism of coupled growth. Some of the effects that are

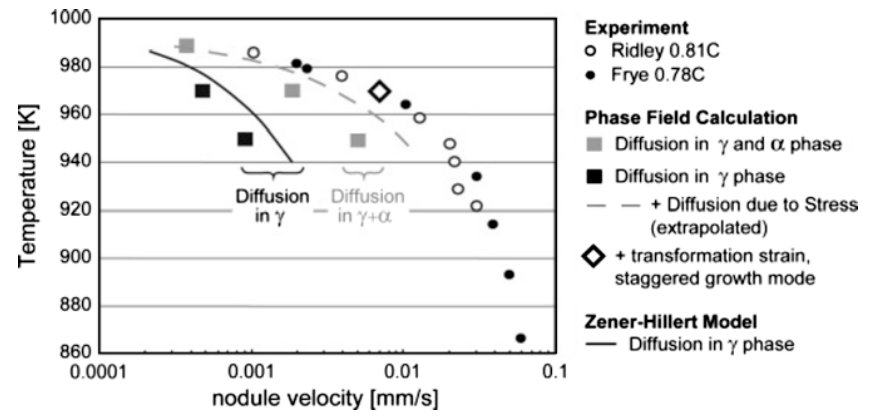

Fig. 18. Comparison of measured and calculated growth temperatures as a function of growth velocity of pearlite. Open and full circles are experimental data; the other symbols are from theory [364].

important in a eutectoid can also become important in a eutectic at high growth rates or in polymer eutectics. For example, the scaling laws, $\lambda^{3} V=$ const. and $\Delta T^{6} \propto V$, have been observed in a polymer system, and it has been shown that accounting for interface diffusion gives the scaling law between the velocity and spacing, while the scaling law for undercooling is controlled by the interface kinetics for secondary nucleation of the molecular attachment process [365].

\subsection{Isothermal transformation}

\subsubsection{Single-phase growth}

Certain solid-state precipitates can be said to be dendritic, as, for example, in the case of the precipitation of $\gamma$ in $\beta$ brass [366] and the formation of $\mathrm{M}_{23} \mathrm{C}_{6}$ carbides in alloy steels [367]. Well-developed dendritic growth has also been observed in glass as it undergoes crystallization $[368,369]$. Sometimes grain boundary-initiated precipitates possess a 2D dendritic morphology. Perhaps more typical is the formation of $\alpha$ needles from $\beta$ brass, a reaction that leads to Widmanstaetten precipitates, which show very little tendency to side branch. However, these precipitates do show approximately paraboloidal fronts near the tip regions, and the side faces are locked into a low energy/ low mobility zone. The analogy with dendrite growth, i.e. the capillarity-corrected Ivantsov model [370,371], has been used to predict lengthening kinetics, with some success [372-374]. Recently, good agreement with measured temperature dependence of growth was found using current phase-field models for 2D dendritic growth [375]. The basic concept of $2 \mathrm{D}$ dendrite tip stability was used to predict the selection of tip radius of Widmanstaetten precipitates at low and high growth rates [376]. The tip selection originating from the constraint due to low mobility sides in this case was found to coincide with the maximum growth rate condition. Since the Peclet number in the solid can be large, rapid transformation effects were also considered, which gave results [376] similar to the Ivantsov marginally stability dendrite model [377].

Several recent studies have used simplified phase-field models to simulate the precipitates' evolution from an ini- 
tial fluctuation $[378,379]$. The formation of Widmanstaetten side plates from prior grain boundary precipitates has been modeled [378], using a highly anisotropic interfacial free energy but neglecting anisotropy of mobility and strain energy. For further progress in this area, we look to a newly developed and highly promising adaptation of the phase-field approach by Greenwood and Provatas [375], which accounts for the interplay of anisotropies of interfacial energy, interfacial mobility and self-strain energy, as well as the strain energy associated with the gradient of a misfitting solute in the parent crystal. Phase-field modeling of various aspects of transformations in $\mathrm{Fe}-\mathrm{C}$ steels, such as grain growth, massive transformation, nucleation and coarsening, has also been undertaken [380,381].

Multi-phase diffusion couples have been used widely in the study of morphological stability in the solid state, particularly for the multicomponent case. Concepts of interfacial stability drawn from solidification theory have found extensive application to evaluate the non-equilibrium effects at the interface that are often critical in ternary steels of composition $\mathrm{Fe}-\mathrm{CX}$, where $\mathrm{X}$ is a substitutional ternary element such as $\mathrm{Ni}, \mathrm{Mn}$ or $\mathrm{Cr}[382,383]$, which may or may not partition at the interface. For example, the observed kinetics are often precisely parabolic and controlled by the diffusion of the interstitial solute. A much more slowly diffusing substitutional solute (e.g. Ni) does not partition, but its near-interface behavior is critical in determining the interfacial boundary conditions for the diffusion of carbon. The method has proven ideal for the study of transitions between full local equilibrium [384] states, for which a pile up of the substitutional solute occurs in the parent phase, and partial local equilibrium ("paraequilibrium" [385]) interfacial states, as well as the investigation of the morphological stability of the transformation interface [386].

In in situ observations of solidification and solid-state transformation of peritectic $\mathrm{Fe}-\mathrm{Ni}$ alloys it was found that the peritectic $\gamma$ phase precipitating at grain boundaries of the supersaturated $\delta$-phase developed morphological instabilities which grew perpendicularly to the grain boundaries $[387,388]$. Solidification theory has been successfully applied for the interpretation of these observations.

In solid-state transformations anisotropy in interface energy and in interface mobility play a dominant role in governing precipitate morphologies. For such cases phase-field models developed for faceted solids may be useful for more quantitative insights.

\subsubsection{Two-phase growth}

In several studies of isothermal growth, the effect of alloying elements on eutectoid growth has been examined. For example, the ideas of Hillert [389] on the effects of Mn additions on the growth of pearlite were tested quantitatively [390].

A solid-state reaction which has no liquid-solid analogue is discontinuous precipitation. In this case, a migrating grain boundary acts as a conduit for solute from the parent phase to a regular array of precipitates [391,392]. Brener and Temkin developed a model that took into account the elastic effects that arise because of the sharp concentration change ahead of the transformation front [393]. They showed that elastic effects are required to obtain a match with the experimentally observed result for realistic values of the physical parameters. The final morphology is very like that expected from a cooperative eutectoid, except that only two phases $\left(\alpha=\alpha^{\prime}+\beta\right)$ participate in the overall reaction. The question whether stability considerations offer a criterion for the selection of the growth velocity and spacing [394] or whether a continuous family of stable steadystate solutions exists [395] is still open.

\subsection{Outlook: solid-state transformation parallels}

While the parallels between liquid-solid and solid-solid phase transformations are many and striking, we must return to, and even stress, the points of divergence, involving the influence of strong anisotropy of interfacial properties and the effects of strain and strain energy on the solid state. These factors restrict the types of transformation that can profitably be taken as analogues. Nevertheless, researchers in the two subdisciplines share many fundamental concerns, especially those dealing with the stability of growth fronts and transformation products.

For the future, we suppose that, by careful selection of systems to study, one can take advantage of the ease of quenching and retaining transformation structures in solid-state transformation products and the potential for high-resolution chemical analysis of the interfacial and near interfacial concentration fields. The absence of convection effects may also be considered a benefit.

There is considerable potential for the use of directional growth experiments for the study of solid-solid reactions as they are superior to isothermal experiments with respect to a control of velocity and temperature gradient and growth under steady-state conditions. The interface composition and interface temperature are quantitatively accessible. At high temperatures, solidification models are sometimes applicable and allow interesting comparisons between solid-liquid and solid-solid growth fronts. In contrast, the effects of strain energy, complex interface kinetics and surface diffusion often play an important role in eutectoid transformations and give rise to different scaling laws between velocity, undercooling and spacing. Directional transformation experiments are therefore capable of providing critical information on the importance of these effects on transformation kinetics. We also emphasize the useful analogy between a temperature gradient in directional solidification experiments and a gradient of fast-diffusing interstitial solute (e.g. C, H, O, N) in isothermal directional experiments like studies of decarburization and internal oxidation.

In future, we expect that experimental research on solidsolid transformations will maintain a primary focus on the results of ultra-high-resolution techniques that yield both 
structural and chemical information [396]. The simulation of these transformations will increasingly involve advanced computationally intensive techniques, such as molecular dynamics [397], phase-field calculations [375], multiscale models [109] and the recently developed phase-field-crystal methods [152]. This latter method resolves the atomic-scale density wave structure of a polycrystalline material and describes its evolution in the presence of elastic interactions on time scales that are orders of magnitude longer than molecular dynamics simulations. Thus, it appears ideally suited to study solid-state phase transformations and promises to become a major modeling technique for the next decades.

Further, we suggest that it would be worthwhile to examine two of the little-explored solid-state transformations: peritectoid and monotectoid systems. Here the absence of convection will be a major advantage for obtaining relevant experimental results.

Finally, a promising approach for the modeling of the properties of cast alloys has to be mentioned. Gandin et al. integrated the modeling of various aspects of phase transformations (solidification, homogenization and precipitation), with structural hardening and mechanical behavior. Their results indicate ways to improve the complete processing chain from the melt to the final heat treated solid [398,399].

\section{Conclusions}

This review shows the progress that has been made in solidification science over the recent years. To summarize, some important challenges and major directions for future research are given. They are subdivided into experimental and computational techniques, and open questions.

Experimental challenges: Direct measurements of interface properties and their anisotropies in real systems are required to confirm the results of atomistic computations. Use of colloidal model systems could improve substantially our understanding of nucleation. Extensive use of 3D imaging techniques [400] and real-time in situ observations will produce new insight into the complex phenomena of growth and defect formation. The quantitative evaluation of microstructures and mechanical properties of the mushy zone, especially at high volume fractions of solid, are important for the understanding of nucleation and growth of second phases, pores and cracks. As fluid flow strongly influences the microstructures, its control and absence in directional solidification by the use of small-diameter tubes is a powerful technique, complementary to space experiments. More work on reliable phase diagrams, which are the basis of any microstructure modeling, is necessary. Inverse methods using microstructure selection maps for the quantitative determination of heterogeneous phase equilibria can be useful in this respect [401]. The highly important field of solidstate transformations can profit from directional transformation experiments, which permit excellent control of a wide range of transformation conditions.
Modeling directions: The integration of molecular dynamics and phase-field techniques, the phase-field crystal method and micro-macro modeling techniques will lead to more realistic predictions of real processes. Enhanced accuracy in the atomistic simulations will require continued efforts aimed at the development of more quantitative interatomic potential models. Three-dimensional modeling and incorporation of fluid flow in these models is necessary. Faster computational algorithms and improved physical models for the calculation of multicomponent phase equilibria and improved thermodynamic data banks are prerequisites for any successful simulation [402]. Atomistic modeling of heterogeneous nucleation and inoculation shows great potential for progress of our understanding of the first moments of phase transformations. Integrated modeling of solidification with solid-state transformations and properties would help process optimization and alloy development.

Open questions: What is the effect of concentration dependent and off-diagonal diffusion terms on diffusional transformation kinetics in multicomponent systems? What are the mechanisms of cell to dendrite and dendrite to cell transitions? How do 3D instabilities affect the operating states of eutectics with anisotropic solid-solid interface energies? Is it really impossible to grow large eutectic single grains? Which microstructure selection mechanisms operate in three-phase eutectics? Which operating states exist in faceted-non-faceted eutectics? What are the mechanisms of eutectic growth transitions from lamellar to fibrous to spherulitic morphologies? Which mechanisms lead to coupled peritectic growth? Does coupled peritectoid growth exist? How does melt convection affect; dendritic growth at high flow velocities, dendritic and eutectic spacings, and dendrite fragmentation? How does stress and deformation affect initiation and growth of pores and cracks in the mushy zone with a high solid fraction? What are the effects of strain energy and anisotropic interface kinetics in solidstate transformations? How can the scaling laws of eutectoid growth be improved? What are the analogies and differences between rapid (high Peclet number) growth in the solid state and rapid solidification?

These are some of the more important questions that spring to mind when analyzing the present understanding of phase transformations. These questions clearly indicate that, despite the substantial progress that has been made in solidification science over the recent years, there is much room left for future contributions to this scientifically and technologically important field.

\section{Acknowledgements}

M.A. and A.K. thank the US Department of Energy for support through Grants DE-FG02-07ER46400 (A.K.), DE-FG02-01ER45910 (M.A.) and DE-FG02-06ER46282 (M.A.), and funds from the Computational Materials Science Network. The work of C.B. was supported, in part, by NASA under Contract NNM04AA18G. 
Appendix A

\begin{tabular}{|c|c|}
\hline \\
\hline \multicolumn{2}{|c|}{$\begin{array}{l}\text { List of symbols } \\
C_{\mathrm{o}} \quad \text { concentration }\end{array}$} \\
\hline$D$ & liquid-phase diffusivity \\
\hline$d_{0}$ & capillary length \\
\hline$g$ & volume fraction \\
\hline$K$ & permeability \\
\hline$k(V)$ & velocity-dependent partition coefficient \\
\hline$k_{\mathrm{B}}$ & Boltzman constant \\
\hline $\mathrm{L}$ & latent heat of melting \\
\hline M & atomic mass \\
\hline$\hat{n}$ & interface normal \\
\hline $\mathrm{P}$ & Peclet number $(R V / 2 D)$ \\
\hline$p$ & pressure \\
\hline$R$ & radius \\
\hline $\mathrm{S}$ & interface stiffness \\
\hline $\mathrm{T}$ & temperature \\
\hline$T_{\text {coh }}$ & coherency temperature \\
\hline$T_{\text {liq }}$ & liquidus temperature \\
\hline$T_{\mathrm{M}}$ & melting temperature \\
\hline$T_{\text {sol }}$ & solidus temperature \\
\hline $\mathrm{t}$ & time \\
\hline V & interface velocity \\
\hline$V_{\mathrm{a}}$ & absolute stability \\
\hline$V_{\mathrm{s}}$ & sound velocity \\
\hline$V_{\mathrm{T}}$ & thermal velocity \\
\hline$x_{\mathrm{L}}$ & solute mole fractions in liquid \\
\hline$x_{\mathrm{S}}$ & solute mole fractions in solid \\
\hline$\alpha$ & Turnbull coefficient \\
\hline$\gamma$ & crystal-melt interfacial free energy \\
\hline$\gamma_{0}$ & orientation-averaged interfacial free energy \\
\hline$\Delta S$ & entropy of melting \\
\hline$\Delta T$ & interface undercooling \\
\hline$\Delta T_{\mathrm{b}}$ & coalescence undercooling \\
\hline$\Delta T_{\mathrm{o}}$ & equilibrium solidification interval \\
\hline$\delta$ & thickness of the solid-liquid interface \\
\hline$\varepsilon_{1}$ & $\begin{array}{l}\text { amplitude of the first term in a cubic } \\
\text { harmonic expansion of } \gamma \text { in } 3 \mathrm{D}\end{array}$ \\
\hline$\varepsilon_{2}$ & $\begin{array}{l}\text { amplitude of the second term in a cubic } \\
\text { harmonic expansion of } \gamma \text { in } 3 \mathrm{D}\end{array}$ \\
\hline$\varepsilon_{4}$ & amplitude of the fourfold variation of $\gamma$ \\
\hline$\varepsilon_{\mathrm{s}}$ & strain \\
\hline$\varepsilon_{\mathrm{k}}$ & kinetic anisotropy \\
\hline$\dot{\varepsilon}_{S}$ & strain rate \\
\hline$\eta$ & viscosity \\
\hline$\theta$ & angle \\
\hline$\mu$ & kinetic coefficient $=V / \Delta T$ \\
\hline$\rho$ & atomic density \\
\hline$\sigma$ & stability parameter \\
\hline$\sigma_{\mathrm{eq}}$ & equivalent von Mises stress \\
\hline$\sigma_{\mathrm{s}}$ & stress tensor in the solid \\
\hline$\phi$ & angle \\
\hline$\varphi$ & angle \\
\hline$v$ & velocity \\
\hline
\end{tabular}

\section{References}

[1] Kurz W, Trivedi R. Acta Metall Mater 1990;38:1.

[2] Boettinger WJ, Coriell SR, Greer AL, Karma A, Kurz W, Rappaz M, et al. Acta Mater 2000;48:43.

[3] Langer JS. In: Souletie J, Vannimenus J, Stora R, editors. Chance and matter, Lectures on the theory of pattern formation, Les Houches, Session XLVI, North Holland; 1987. p. 629.

[4] Kessler D, Koplik J, Levine H. Adv Phys 1988;37:255.

[5] Karma A, Rappel W-J. Phys Rev E 1998;57:4323.

[6] Liu S, Napolitano RE, Trivedi R. Acta Mater 2001;49:4271.

[7] Napolitano RE, Liu S, Trivedi R. Interface Sci 2002;10:217.

[8] Napolitano RE, Liu S. Phys Rev B 2004;70:214103.

[9] Passerone A, Eustathopoulos N. Acta Mater 1982;30:1349.

[10] Glicksman ME, Schaefer RJ. J Cryst Growth 1967;1:297.

[11] Rodway GH, Hunt JD. J Cryst Growth 1991;112:554.

[12] Hoyt JJ, Asta M, Karma A. Mater Sci Eng R 2003;41:121.

[13] Hoyt JJ, Asta M, Haxhimali T, Karma A, Napolitano RE, Trivedi R, et al. MRS Bull 2004;29:935.

[14] Davidchack RL, Laird BB. J Phys Chem B 2005;109:17802.

[15] Frenkel D, Smit B. Understanding molecular simulation: from algorithms to applications. San Diego (CA): Academic Press; 2002.

[16] Daw MS, Baskes MI. Phys Rev Lett 1983;50:1285.

[17] Daw MS, Baskes MI. Phys Rev B 1984;29:6443.

[18] Turnbull D. J Appl Phys 1950;21:1022.

[19] Kelton KF. Solid State Phys 1991;45:75.

[20] Granasy L, Borzsonyi T, Pusztai T. Phys Rev Lett 2002;88: 206105.

[21] Waseda Y, Miller WA. Trans Jpn Inst Metals 1978;19:546.

[22] Glicksman ME, Vold CL. Acta Metall 1969;17:1.

[23] Glicksman ME, Vold CL. Scr Metall 1983;5:493.

[24] Eustathopoulos N. Int Met Rev 1983;28:189.

[25] Spaepen F. Acta Metall Mater 1975;23:729.

[26] Spaepen F, Mayer RB. Scr Metall Mater 1976;10:257.

[27] Thompson CV. Ph.D. thesis, Harvard University; 1979.

[28] Herlach DM, Galenko P, Holland-Moritz D. Metastable solids from undercooled melts. Oxford: Pergamon; 2007.

[29] Cech RE. Trans AIME 1956;206:535.

[30] Ghosh G. Mater Sci Eng A 1994;189:277.

[31] Volkmann T, Loeser W, Herlach DN. Metall Mater Trans A 1997;28A:453.

[32] Zambon A, Badan B, Eckler K, Gärtner F, Norman AF, Greer AL, et al. Acta Mater 1998;46:4657.

[33] Li M, Lin Z, Song G, Yang G, Zhou Y. Mater Sci Eng A 1999;268:90.

[34] Herlach DM. J Phys Condens Matter 2001;13:7737.

[35] Greer AL, Walker IR. J Non-Cryst Solids 2003;317:78.

[36] Notthoff C, Feuerbacker V, Franz H, Herlach DM, Holland-Moritz D. Phys Rev Lett 2001;86:1038.

[37] Holland-Moritz D. Int J Non-Equilib Process 1998;11:169.

[38] Holland-Moritz D. J Non-Cryst Solids 1999;252:839.

[39] Holland-Moritz D. Mater Sci Eng A 2001;304-306:108.

[40] Holland-Moritz D, Herlach DM, Grushko B, Urban K. Mater Sci Eng A 1994;182:766.

[41] Schroers J, Holland-Moritz D, Herlach DM, Urban K. Phys Rev B 2000;61:14500.

[42] Broughton JQ, Gilmer GH. J Chem Phys 1986;84:5759.

[43] Hoyt JJ, Asta M, Sun DY. Philos Mag 2006;86:3651.

[44] Sun DY, Asta M, Hoyt JJ, Mendelev MI, Srolovitz DJ. Phys. Rev. B 2004;69:020102.

[45] Davidchack RL, Morris JR, Laird BB. J Chem Phys 2006;125:094710.

[46] Morris JR, Mendelev MI, Srolovitz DJ. J Non-Cryst Solids 2007;353:3565.

[47] Fehlner WR, Vosko SH. Can J Phys 1976;54:2159.

[48] Hoyt JJ, Asta M, Karma A. Phys Rev Lett 2001;86:5530.

[49] Morris JR. Phys Rev B 2002;66:144104. 
[50] Haxhimali T, Karma A, Gonzales F, Rappaz M. Nat Mater 2006;5:660.

[51] Becker CA, Olmsted D, Asta M, Hoyt JJ, Foiles SM. Phys Rev Lett 2007;98:125701.

[52] Davidchack RL, Laird BB. Phys Rev Lett 2005;94:086102.

[53] Morris JR, Song X. J Chem Phys 2003;119:3920.

[54] Davidchack RL, Laird BB. J Chem Phys 2003;118:7651.

[55] Davidchack RL, Laird BB. Phys Rev Lett 2000;85:4751.

[56] Gonzales F, Rappaz M. Metall Trans A 2006;37:2797.

[57] Jackson KA. Interface Sci 2002;10:159.

[58] Chernov AA. Modern crystallography, vol. 3. Berlin: Springer; 1984.

[59] Aziz MJ, Boettinger WJ. Acta Metall Mater 1994;42:527.

[60] Boettinger WJ, Aziz MJ. Acta Metall 1989;37:3379.

[61] Turnbull D, Bagley BG. In: Hannay NG, editor. Treatise on solid state chemistry, vol. 5. New York: Plenum; 1975. p. 526.

[62] Coriell SR, Turnbull DH. Acta Metall 1982;30:2135.

[63] Broughton JQ, Gilmer GH, Jackson KA. Phys Rev Lett 1982;49:1496

[64] Hoyt JJ, Sadigh B, Asta M, Foiles SM. Acta Mater 1999;47:3181.

[65] Mikheev LV, Chernov AA. J Cryst Growth 1991;112:591.

[66] Chernov AA. J Cryst Growth 2004;264:499.

[67] Burke E, Broughton JQ, Gilmer GH. J Chem Phys 1988;89:1030.

[68] Tepper HL, Briels WJ. J Chem Phys 2002;116:5186.

[69] Tepper HL, Briels WJ. J Chem Phys 2001;115:9434.

[70] Huitema HEA, Vlot MJ, van der Eerden JP. J Chem Phys 1999;111:4714.

[71] Amini M, Laird BB. Phys Rev Lett 2006;97:216102.

[72] Hoyt JJ, Asta M, Karma A. Interface Sci 2002;10:181.

[73] Laird BB. J Chem Phys 2001;115:2887.

[74] Perepezko JH. In: Rappaz M, Beckermann C, Trivedi R, editors. Solidification processes and microstructures. A symposium in honor of Wilfried Kurz. Warrendale (PA): TMS; 2004. p. 295.

[75] Greer AL, Quested TE. Philos Mag 2006;86:3665.

[76] Kelton KF, Greer AL, Herlach DM, Holland-Moriz D. MRS Bull 2004;29:940.

[77] ten Wolde PR, Ruiz-Montero MJ, Frenkel D. J Chem Phys 1999;110:1591.

[78] Auer S, Frenkel D. Nature 2001;409:1020.

[79] Auer S, Frenkel D. Annu Rev Phys Chem 2004;55:333.

[80] Trudu F, Donadio D, Parrinello M. Phys Rev Lett 2006;97:105701.

[81] Streitz FH, Glosli JN, Patel MV. Phys Rev Lett 2006;96:225701.

[82] Desgranges C, Delhommelle J. J Chem Phys 2007;126:054501.

[83] Aga RS, Morris JR, Hoyt JJ, Mendelev M. Phys Rev Lett 2006;96:245701.

[84] Huitema HEA, ven der Eerden JP, Janssen JJM, Human H. Phys Rev B 2000;62:14690.

[85] Wu DT, Granasy L, Spaepen F. MRS Bull 2004;29:945.

[86] Spaepen F. Solid State Phys 1994;47:1.

[87] Bai XM, Li M. J Chem Phys 2006;124:124707.

[88] Gasser U, Weeks ER, Schofield A, Pusey PN, Weitz DA. Science 2001:292:258.

[89] Gránásy L, Börzsönyi T, Pusztai T. Phys Rev Lett 2002;88:206105.

[90] Gránásy L, Pusztai T. J Chem Phys 2002;117:10121.

[91] Tóth G, Gránásy L. J Chem Phys 2007;127:074709.

[92] Gránásy L, Pusztai T, Saylor D, Warren JA. Phys Rev Lett 2007;98:035703

[93] ten Wolde PR, Ruiz-Montero MJ, Frenkel D. J Chem Phys 1996;104:9932.

[94] Auer S, Frenkel D. J Phys Condens Matter 2002;14:7667.

[95] Browning AR, Doherty MF, Fredrickson GH. Phys Rev E 2008;77:041604.

[96] Desgranges C, Delhommelle J. Phys Chem Phys 2007;127:144509.

[97] Wang H, Gould H, Klein W. Phys Rev E 2007;76:031604.

[98] Ashkenazy Y, Averback RS. Europhys Lett 2007;79:26005.

[99] Aziz MJ, Kaplan J. Acta Metall 1988;36:2335.

[100] Aziz MJ. Metall Mater Trans A 1996;27:671.

[101] Jackson KA, Beatty KM, Gudgel KA. J Cryst Growth 2004;271:481.
[102] Galenko PK, Danilov DA. J Cryst Growth 1999;197:992.

[103] Ahmad NA, Wheeler AA, Boettinger WJ, McFadden GB. Phys Rev E 1998:58:3436.

[104] Cai Y, Zhang G, Fu H. J Appl Phys 2000;87:7735.

[105] Danilov D, Nestler B. Acta Mater 1996;54:4659.

[106] Kerrache A, Horbach J, Binder K. Europhys Lett 2008;81:58001.

[107] Sluiter M, Kawazoe Y. Phys Rev B 1996;54:10381.

[108] Asta M, Ozolins V, Woodward C. JOM 2001;53:16

[109] Vaithyanathan V, Wolverton C, Chen LQ. Phys Rev Lett 2002;88:125503.

[110] Hafner J, Wolverton G, Ceder G. MRS Bull 2006;31:659.

[111] Bi ZQ, Sekerka RF. J Cryst Growth 2002;237:138.

[112] Kauerauf B, Zimmermann G, Rex S, Billia B, Jamgotchian H, Hunt JD. J Cryst Growth 2001;223:277.

[113] Karma A. Phys Rev Lett 2001;87:115701.

[114] Ramirez JC, Beckermann C, Karma A, Diepers H-J. Phys Rev E 2004;69:051607.

[115] Echebarria B, Folch R, Karma A, Plapp M. Phys Rev E 2004;70:061604.

[116] Provatas N, Goldenfeld N, Dantzig J, LaCombe JC, Lupulescu A, Koss MB, et al. Phys Rev Lett 1999;82:4496.

[117] Plapp M, Karma A. Phys Rev Lett 2000;84:1740.

[118] Fan J, Greenwood JM, Haataja M, Provatas N. Phys Rev E 2006;74:031602.

[119] Bragard J, Karma A, Lee YH, Plapp M. Interface Sci 2002;10:121.

[120] Greenwood M, Haataja M, Provatas N. Phys Rev Lett 2004;93:246101.

[121] Barbieri A, Langer JS. Phys Rev A 1989;39:5314.

[122] Ben Amar M, Brener EA. Phys Rev Lett 1993;71:589.

[123] Chan S-K, Reimer H-H, Kahlweit M. J Cryst Growth 1976;32: 303.

[124] Liu S, Li J, Lee J, Trivedi R. Philos Mag 2006;86:3717.

[125] Haxhimali T. Ph.D. thesis, Northeastern University; 2006

[126] Nestler B, Danilov D, Galenko R. J Comput Phys 2005;207:221.

[127] Lee YH. Ph.D. thesis, Northeastern University; 1998.

[128] Mullis AM, Cochrane RF. Acta Mater 2001;49:2205.

[129] Boettinger WJ, Warren JA. J Cryst Growth 1999;200:583.

[130] Danilov D, Nestler B. Phys Rev Lett 2004;93:215501.

[131] Brener EA, Muller-Krumbhaar H, Temkin DE. Phys Rev E 1996;54:2714.

[132] Abel T, Brener EA, Muller-Krumbhaar H. Phys Rev E 1997;55:7789.

[133] Pettersen K, Ryum M. Metall Trans A 1989;20:847.

[134] Pettersen K, Lohne O, Ryum M. Metall Trans A 1990;21:221.

[135] Henry S, Jarry P, Jouneau P-H, Rappaz M. Metall Mater Trans A 1997;28:207.

[136] Henry S, Jarry P, Rappaz M. Metall Mater Trans A 1998;29:2807.

[137] Henry S, Minghetti T, Rappaz M. Acta Mater 1998;46:6431.

[138] Henry S. PhD thesis, EPFL Lausanne; 1999.

[139] Semoroz A, Durandet Y, Rappaz M. Acta Metall Mater 2001:49:529.

[140] Semoroz A. Ph.D. thesis, EPFL Lausanne; 1999.

[141] Koss MB, LaCombe JC, Chait A, Pines V, Zlatkowski M, Glicksman ME, et al. J Cryst Growth 2005;279:170.

[142] Diepers H-J, Karma A. In: Rappaz M, Beckermann C, Trivedi R, editors, Solidification processes and microstructures. A symposium in honor of Wilfried Kurz. Warrendale (PA): TMS; 2004. p. 369-71.

[143] Martinez RA, Karma A, Flemings MC. Metall Mater Trans A 2006;37:2807.

[144] Uehara T, Sekerka RF. J Cryst Growth 2003;254:251.

[145] Debierre J-M, Karma A, Celestini F, Guérin R. Phys Rev E 2003;68:041604.

[146] Greenwood M, Haataja M, Provatas AN. Phys Rev Lett 2004;93:246101.

[147] Plapp M, Karma A. Phys Rev E 2002;66:061608.

[148] Zhang R, Jing T, Jie W, Baicheng L. Acta Mater 2006;54:2235.

[149] Tong Chaohui, Greenwood M, Provatas N. Phys Rev B 2008;77:064112. 
[150] Gránásy L, Pusztai T, Warren JA, Douglas JF, Börzsönyi T, Ferreiro V. Nat Mater 2003;2:92.

[151] Gránásy L, Pusztai T, Börzsönyi T, Warren JA, Douglas JF. Nat Mater 2004;3:645.

[152] Provatas N, Dantzig JA, Athreya B, Chan P, Stefanovic P, Goldenfeld N, et al. JOM 2007;59:83.

[153] Wu K-A, Karma A. Phys Rev B 2007;76:184107.

[154] Karma A, Plapp M. J Metals 2004;56:28.

[155] Akamatsu S, Plapp M, Faivre G, Karma A. Metall Mater Trans A 2004;35:1815

[156] Jackson KA, Hunt JD. Trans AIME 1966;236:1129.

[157] Langer JS. Phys Rev Lett 1980;44:1023.

[158] Trivedi R, Liu S, Mazumder P, Simsek E. Sci Technol Adv Mater 2001;2:309.

[159] Walker H, Liu S, Lee JH, Trivedi R. Metall Mater Trans A 2007;38:1417

[160] Akamatsu S, Bottin-Rousseau S, Faivre G. Phys Rev Lett 2004;93:175701.

[161] Akamatsu S, Bottin-Rousseau S, Faivre G. Philos Mag 2006;86:3703.

[162] Akamatsu S, Bottin-Rousseau S, Perrut M, Faivre G, Witusiewicz VT, Sturz L. J Cryst Growth 2007;299:418.

[163] Parisi A, Plapp M. Acta Mater 2008;56:1348.

[164] Teng J, Liu S, Trivedi R. Acta Mater 2008;56:2819.

[165] Serefoglu M, Napolitano RE. Acta Mater 2008;56:3862.

[166] Liu S, Lee JH, Enlow D, Trivedi R. In: Rappaz M, Beckermann C, Trivedi R, editors. Solidification processes and microstructures. A symposium in honor of Wilfried Kurz. Warrendale (PA): TMS; 2004. p. 257.

[167] Wang N, David S, Walker H, Trivedi R. Trans Indian Inst Metals 2007;60:69.

[168] Trivedi R, Magnin P, Kurz W. Acta Metall 1987;35:971.

[169] Akamatsu S, Faivre G. Phys Rev E 2000;61:3757.

[170] Han SH, Trivedi R. Unpublished data, Iowa State University, Ames, IA; 2008 .

[171] Hecht U, Witusiewicz VT, Drevermann A, Bottger B, Rex S. Mater Sci Forum 2005;508:57.

[172] Rex S, Bottger B, Witusiewicz V, Hecht U. Mater Sci Eng A 2005;413:249.

[173] Witusiewicz VT, Hecht U, Sturz L, Rex S. J Cryst Growth 2006;286:137.

[174] Witusiewicz VT, Hecht U, Sturz L, Rex S. J Cryst Growth 2006;286:431.

[175] Witusiewicz VT, Hecht U, Sturz L, Rex S. J Cryst Growth 2006;297:117

[176] Fisher DJ, Kurz W. Metall Trans 1974;5:1508.

[177] Himemiya T, Umeda T. JIM 1999;40:665.

[178] Hecht U, Gránásy L, Pusztai T, Böttger B, Apel M, Witusiewicz V, et al. Mater Sci Eng R-Rep 2004;46:1.

[179] Lee JH, Verhoeven JD. J Cryst Growth 1994;144:353.

[180] Vandyoussefi M, Kerr HW, Kurz W. Acta Mater 2000;48:2297.

[181] Boettinger WJ. Metall Trans 1974;5:2023.

[182] Mazumder P, Trivedi R. Phys Rev Lett 2002;88:235507.

[183] Trivedi R, Park JS. J Cryst Growth 2002;235:572.

[184] Lograsso TA, Fuh BC, Trivedi R. Metall Mater Trans A 2005;36:1287.

[185] Trivedi R, Shin JH. Mater Sci Eng A 2005;413/14:288.

[186] Lo TS, Dobler S, Plapp M, Karma A. Acta Mater 2003;51:599.

[187] Dobler S, Lo TS, Plapp M, Karma A. Acta Mater 2004;52:2795.

[188] Kohler F. Ph.D. thesis, EPFL Lausanne; 2008.

[189] Akamatsu S, Moulinet S, Faivre G. Metall Mater Trans A 2001;32:2039.

[190] Guzik E, Kopycinski D. Metall Mater Trans A 2006;37:3057.

[191] Magnin P, Kurz W. Acta Metall 1987;35:1119.

[192] Lasagni F, Lasagni A, Holzapfel C, Muecklich F, Degischer HP. Adv Eng Mater 2006;8:719.

[193] Napolitano RE, England LG. In: Solidification of aluminum alloys. Charlotte (NC): TMS; 2004.
[194] Shamsuzzoha M, Hogan LM. Cast Metals 1991;4:103.

[195] Napolitano RE, Meco H, Jung C. J Metals 2004;56:16.

[196] Nogita K, Dahle AK. Mater Characterization 2001;46:305.

[197] Dahle AK, Nogita K, Zindel JW, McDonald SD, Hogan LM. Metall Mater Trans A 2001;32:949.

[198] McDonald SD, Dahle AK, Taylor JA, St. John DH. Metall Mater Trans A 2004;35:1829.

[199] McDonald SD, Nogita K, Dahle AK. Acta Mater 2004;52:4273.

[200] Dahle AK, Nogita K, McDonald SD, Dinnis C, Lu L. Mater Sci Eng A 2005;413:243.

[201] Shankar S, Riddle YW, Makhlouf MM. Metall Mater Trans A 2004;35:3038.

[202] Shankar S, Riddle YW, Makhlouf MM. Metall Mater Trans A 2005;36:1613

[203] Dahle AK, Hillert M. Metall Mater Trans A 2005;36:1612.

[204] Dahle AK, Hillert M. Metall Mater Trans A 2006;37:1353.

[205] Shankar S, Makhlouf MM. Metall Mater Trans A 2006;37:1353.

[206] Eggleston JJ, McFadden GB, Voorhees PW. Physica D 2001;150: 91.

[207] Debierre J-M, Karma A, Celestini F, Guerin R. Phys Rev E 2003;68:041604.

[208] Suzuki T, Kim SG, Kim WT. Mater Sci Eng A 2007;449-451:99.

[209] Perepezko JH, Hildal K. Philos Mag 2006;86:3681.

[210] Gránásy L, Pusztai T, Börzsönyi T, Tóth GI, Tegze G, Warren JA, et al. Philos Mag 2006;86:3757.

[211] Buehler L, Davis SH. J Cryst Growth 1998;186:629.

[212] Chen YJ, Davis SH. J Fluid Mech 2000;421:369.

[213] Chen YJ, Davis SH. J Fluid Mech 1999;395:253.

[214] Jamgotchian H, Bergeon N, Benielli D, Voge P, Billia B, Guerin R. Phys Rev Lett 2001;87:166105.

[215] Jamgotchian H, Nguyen-Thi H, Bergeon N, Billia B. Int J Thermal Sci 2004;43:769.

[216] Noel N, Jamgotchian H, Billia B. J Cryst Growth 1998;187:516.

[217] Lan CW, Tu CY. J Cryst Growth 2000;220:619.

[218] Lan CW, Yang YW, Chen HZ. Metall Mater Trans A 2002;33:3011.

[219] Lan CW, Lee MH, Chuang MH, Shih CJ. J Cryst Growth 2006;295:202.

[220] Kauerauf B, Zimmermann G, Murmann L, Rex S. J Cryst Growth 1998;193:701.

[221] Glicksman ME, Huang S-C. In: Zierip J, Oertl Jr H, editors. Convective transport and instability phenomena. Karlsruhe: G. Braun; 1982. p. 557.

[222] Glicksman ME, Coriell SR, McFadden GB. Annu Rev Fluid Mech 1986; 18:307.

[223] Beckermann C, Diepers HJ, Steinbach I, Karma A, Tong X. J Comput Phys 1999;154:468.

[224] Anderson DM, McFadden GB, Wheeler AA. Physica D 2001;151:305.

[225] Anderson DM, McFadden GB, Wheeler AA. Physica D 2000; $135: 175$

[226] Ai X, Shu Y, Li BQ. Comput Math Appl 2006;52:721.

[227] Galenko PK, Funke O, Wang J, Herlach DM. Mater Sci Eng A 2004;375-377:488.

[228] Galenko PK, Herlach DM, Phanikumar G, Funke O. Mater Sci Forum 2006;508:431

[229] Jeong JH, Dantzig JA, Goldenfeld N. Metall Mater Trans A 2003;34:459.

[230] Lan CW, Hsu CM, Liu CC. J Cryst Growth 2002;241:379.

[231] Lan CW, Shih CJ. Phys Rev E 2004;69:31601.

[232] Lan CW, Shih CJ. J Cryst Growth 2004;264:472.

[233] Lu Y, Beckermann C, Ramirez JC. J Cryst Growth 2005;280:320.

[234] Narski J, Picasso M. Fluid Dyn Mater Proc 2007;3:49.

[235] Tong X, Beckermann C, Karma A, Li Q. Phys Rev E 2001;63:061601.

[236] Tonhardt R, Amberg G. J Cryst Growth 2000;213:161.

[237] Al-Rawahi N, Tryggvason G. J Comput Phys 2002;180:471.

[238] Udaykumar HS, Marella S, Krishnan S. Int J Heat Mass Transfer 2003;46:2615 
[239] Zhao P, Heinrich JC, Poirier DR. Int J Numer Methods Fluids 2005;49:233.

[240] Chakraborty S, Chatterjee D. Phys Lett A 2006;351:359.

[241] Medvedev D, Kassner K. Phys Rev E 2005;72:56703.

[242] Medvedev D, Kassner K. J Cryst Growth 2005;275:1495.

[243] Miller W, Rasin I, Succi S. Physica A 2006;362:78.

[244] Li D, Li R, Zhang P. Appl Math Model 2007;31:971.

[245] Shin YH, Hong CP. ISIJ Int 2002;42:359.

[246] Zhu MF, Lee SY, Hong CP. Phys Rev E 2004;69:061610.

[247] Das A, Ji S, Fan Z. Acta Mater 2002;50:4571.

[248] Bouissou Ph, Pelce P. Phys Rev A 1989;40:6673.

[249] Schrage DS. J Cryst Growth 1999;205:410.

[250] Li Q, Beckermann C. J Cryst Growth 2002;236:482.

[251] Lee J, Liu S, Miyahara H, Trivedi R. Metall Mater Trans B 2004;35:909.

[252] Appolaire B, Albert V, Combeau H, Lesoult G. Acta Mater 1998;46:5851.

[253] Appolaire B, Albert V, Combeau H, Lesoult G. ISIJ Int 1999;39:263.

[254] Badillo A, Ceynar DL, Beckermann C. J Cryst Growth 2007;309:197.

[255] Chen H, Chen YS, Wu X, Tewari SN. J Cryst Growth 2003;253:413

[256] Drevet B, Nguyen-Thi H, Camel D, Billia B, Dupouy MD. J Cryst Growth 2000;218:419.

[257] Hui J, Tiwari R, Wu X, Tewari SN, Trivedi R. Metall Mater Trans A 2002;33:3499.

[258] Kauerauf B, Zimmermann G, Rex S. Mater Sci Forum 2000;329330:271.

[259] Ming L, Mori T, Iwasaki H. Mater Sci Eng A 1999;265:217.

[260] Nguyen-Thi H, Dabo Y, Drevet B, Dupouy MD, Camel D, Billia B, et al. J Cryst Growth 2005;281:654.

[261] Spinelli JE, Rosa DM, Ferreira IL, Garcia A. Mater Sci Eng A 2004:383:271.

[262] Trivedi R, Mazumder P, Tewari SN. Metall Mater Trans A 2002;33:3763.

[263] Trivedi R, Miyahara H, Mazumder P, Simsek E, Tewari SN. J Cryst Growth 2001;222:365.

[264] Apel M, Diepers HJ, Steinbach I. In: Gandin CA, Bellet M, editors. Modeling of casting, welding and adv. solidification processes XI. Warrendale (PA): TMS; 2006. p. 505-12.

[265] Diepers HJ, Steinbach I. Mater Sci Forum 2006;508:145.

[266] Natsume Y, Ohsasa K, Narita T. Mater Trans 2002;43:2228.

[267] Nomoto S, Izumi T, Shiohara Y, Umeda T. J Jpn Inst Metals 2001;65:310

[268] Hainke M, Steinbach S, Dagner J, Ratke L, Muller G. Mater Sci Forum 2006;508:199.

[269] Hainke M, Steinbach S, Ratke L, Muller G. Trans Indian Inst Metals 2005;58:639.

[270] Ratke L, Steinbach S, Muller G, Hainke M, Roosz A, Fautrelle Y, et al. Mater Sci Forum 2006;508:131.

[271] Steinbach S, Ratke L. Mater Sci Eng A 2005;413-414:200.

[272] Lehmann P, Moreau R, Camel D, Bolcato R. Acta Mater 1998:46:4067.

[273] Lehmann P, Moreau R, Camel D, Bolcato R. J Cryst Growth 1998;183:690

[274] Diepers HJ, Beckermann C, Steinbach I. Acta Mater 1999;47:3663

[275] Li X, Ren ZM, Fautrelle Y. J Cryst Growth 2006;290:571.

[276] Li X, Fautrelle Y, Ren Z. Acta Mater 2007;55:1377.

[277] Corrigan DP, Koss MB, LaCombe JC, de Jager KD, Tennenhouse LA, Glicksman ME. Phys Rev E 1999;60:7217.

[278] Giummarra C, LaCombe JC, Koss MB, Frei JE, Lupulescu A, Glicksman ME. J Cryst Growth 2005;274:317.

[279] McCay MH, Hopkins JA, McCay TD. Metall Mater Trans A 1998;29:1137.

[280] Henry S, Gruen GU, Rappaz M. Metall Mater Trans A 2004;35:2495.

[281] Nguyen-Thi H, Reinhart G, Zhou B, Billia B, Liu Q, Lyubimova TP, et al. J Cryst Growth 2005;275:1579.
[282] Badillo A, Beckermann C. Acta Mater 2006;54:2015.

[283] Nguyen-Thi H, Reinhart G, Mangelinck-Noel N, Jung H, Billia B, Schenk T, et al. Metall Mater Trans A 2007;38:1458.

[284] Ruvalcaba D, Mathiesen RH, Eskin DG, Arnberg L, Katgerman L. Acta Mater 2007;55:4287.

[285] Campanella T, Charbon C, Rappaz M. Metall Mater Trans A 2004;35:3201.

[286] Li T, Lin X, Huang W. Acta Mater 2006;54:4815.

[287] Ma D, Jie WQ, Li Y, Ng SC. Acta Mater 1998;46:3203.

[288] Wang WM, Liu JM, Chen XY, Liu ZG. J Cryst Growth 2002;240:313.

[289] Weiqiang Z, Hua F, Yuansheng Y, Zhuangqi H. J Cryst Growth 1998;194:263.

[290] Regel LL, Wilcox WR, Popov D, Li F. Acta Astronaut 2001:48:101.

[291] Lee JH, Liu S, Trivedi R. Metall Mater Trans A 2005;36:3111.

[292] Coriell SR, McFadden GB, Mitchell WF, Murray BT, Andrews JB, Arikawa Y. J Cryst Growth 2001;224:145.

[293] Liu S, Trivedi R. Metall Mater Trans A 2006;37:3293.

[294] Mazumder P, Trivedi R. Appl Math Model 2004;28:109.

[295] Mazumder P, Trivedi R, Karma A. Metall Mater Trans A 2000;31:1233.

[296] Siquieri R, Emmerich H. Philos Mag Lett 2007;87:829.

[297] Nestler B, Wheeler AA, Ratke L, Stocker C. Physica D 2000;141:133.

[298] Ratke L. Metall Mater Trans A 2003;34:449.

[299] Stocker C, Ratke L. J Cryst Growth 2000;212:324.

[300] Yang S, Liu W, Jia J. J Mater Sci 2001;36:5351.

[301] Alkemper J, Voorhees PW. J Microsc 2001;201:388

[302] Alkemper J, Mendoza R, Voorhees PW. Adv Eng Mater 2002;4: 694.

[303] Mendoza R, Alkemper J, Voorhees PW. Z Metallkd 2005;96:155.

[304] Ludwig O, DiMichiel M, Salvo L, Suéry M, Falus P. Metall Mater Trans A 2005;36:1515.

[305] Limodin N, Salvo L, Suéry M, DiMichiel M. Acta Mater 2007;55:3177.

[306] Li B, Brody HD, Kazimirov A. Phys Rev E 2004;70:062602.

[307] Mathiesen RH, Arnberg L, Ramsøskar K, Weitkamp T, Rau C, Snigirev A. Metall Mater Trans B 2002;33:613.

[308] Schenk T, Gastaldi J, Nguyen Thi H, Reinhart G, Mangelinck-Noel N, Klein H, et al. J Cryst Growth 2005;275:201.

[309] Rowenhorst DJ, Kuang JP, Thornton K, Voorhees PW. Acta Mater 2006;54:2027.

[310] Krill III CE, Chen LQ. Acta Mater 2002;50:3057.

[311] Mendoza R, Thornton K, Savin I, Voorhees PW. Acta Mater 2006;54:743.

[312] Kammer D, Voorhees PW. Acta Mater 2006;54:1549.

[313] Smith CS. Trans Metall Soc AIME 1948;175:15.

[314] Rappaz M, Jacot A, Boettinger WJ. Metall Mater Trans A 2003;34:467.

[315] Karma A, private communication

[316] Vernède S, Rappaz M. Philos Mag 2006;86:3779.

[317] Vernède S, Jarry P, Rappaz M. Acta Mater 2006;53:4023.

[318] Rappaz M, Drezet JM, Mathier V, Vernède S. Mater Sci Forum 2006;519-521:1665.

[319] Ni J, Beckermann C. Metall Mater Trans B 1991;22:349.

[320] Stangeland A, Mo A, Nielsen O, Eskin D, M'Hamdi M. Metall Mater Trans A 2004;35:2803.

[321] Stangeland A, Mo A, Eskin D. Metall Mater Trans A 2006;37:2219.

[322] Ludwig O, Drezet JM, Martin CL, Suéry M. Metall Mater Trans A 2005;36:1525.

[323] Ludwig O, Drezet JM, Ménésès P, Martin CL, Suéry M. Mater Sci Eng A 2005;413-414:174.

[324] M'Hamdi M, Mo A, Martin CL. Metall Mater Trans A 2002;33:2081.

[325] Campbell J. Castings. Oxford: Elsevier; 2003.

[326] Lee PD, Chirazi A, See D. J Light Metals 2001;1:15.

[327] Sung PK, Poirier DR, Felicelli SD. Model Simul Mater Sci Eng 2002;10:551 
[328] Péquet C, Gremaud M, Rappaz M. Metall Mater Trans A 2002;33:2095.

[329] Stefanescu DM. Int J Cast Metals Res 2005;18:129.

[330] Carlson KD, Lin Z, Beckermann C. Metall Mater Trans B 2007;38:541.

[331] Wang JS, Lee PD. Int J Cast Metal Res 2007;20:151.

[332] Piwonka TS, Flemings MC. Trans AIME 1966;236:1157.

[333] Feurer U. Giessereiforschung 1976;28:75.

[334] Clyne TW, Davies G. J Brit Foundry 1981;74:65.

[335] Suyitno, Kool WH, Katgermann L. Metall Mater Trans A 2005;36:1537.

[336] Eskin DG, Suyitno, Katgerman L. Prog Mater Sci 2004;49:629.

[337] Lahaie D, Bouchard M. Metall Mater Trans B 2001;32:697.

[338] Magnin B, Maenner L, Katgermann L, Engler S. Mater Sci Forum 1996;217-222:1209.

[339] Rappaz M, Drezet JM, Gremaud M. Metall Mater Trans A 1999;30:449.

[340] Niyama E, Uchida T, Morikawa M, Saito S. AFS Int Cast Metals J 1982:52.

[341] Drezet JM, Rappaz M. In Light Metals. Warrendale (PA): TMS; 2001. p. 887.

[342] Mathier V, Drezet JM, Rappaz M. Model Simul Mater Sci Eng 2007; 15:121.

[343] Wang N, Mokadem S, Rappaz M, Kurz W. Acta Mater 2004;52:3173.

[344] Phillion AB, Cockcroft SL, Lee PD. Acta Mater 2008;4:55.

[345] Zener C. Trans AIME 1947;167:550.

[346] Hillert M. Jernkont Annal 1957;141:757.

[347] Trivedi RK. Metall Trans 1970;1:921.

[348] Hillert M. Acta Mater 1999;47:4481.

[349] Aaronson HI, Furuhara T, Hall MG, Hirth JP, Nie J-F, Purdy GR, et al. Acta Mater 2006;54:1227.

[350] Chen LQ. Annu Rev Mater Res 2002;32:113.

[351] Elder KR, Katakowski M, Haataja M, Grant M. Phys Rev Lett 2002;88:245701.

[352] Trivedi R, Kurz W. Int Mater Rev 1994;39:49.

[353] Kurz W. Adv Mater Eng 2001;3:443.

[354] Vandyoussefi M, Kerr HW, Kurz W. Acta Mater 1997;45:4093.

[355] Lima M, Kurz W. Metall Mater Trans A 2002;33:2337.

[356] Mullins W, Sekerka RF. J Appl Phys 1964;35:444.

[357] Sumida M, Kurz W. EPFL, unpublished; 2001.

[358] Pearson DD, Verhoeven JD. Metall Trans A 1984;15:1037.

[359] Verhoeven JD, Pearson DD. Metall Trans A 1984;15:1947.

[360] Whiting MJ. Scr Mater 2000;43:969.

[361] Trivedi RK. Unpublished work.

[362] Carpay FMA. Int Met Rev 1978;23:1.

[363] Teng J, Liu S, Trivedi R. Acta Mater 2008;56:2819.

[364] Steinbach I, Apel M. Acta Mater 2007;55:4817.

[365] Teng J, Ph.D. Thesis, Ames, IA: Iowa State University; 2007.

[366] Malcolm JA, Purdy GR. TMS AIME 1967;239:1391.
[367] Habraken L, Brouwer JL. De ferri metallographia. Brussels: Presses Academique Europeenes; 1966.

[368] Trivedi RK, Laorchan V. Acta Metall 1988;36:1941.

[369] Trivedi RK, Laorchan V. Acta Metall 1988;36:1951.

[370] Trivedi RK. Scr Metall 1969;3:613.

[371] Trivedi RK. Acta Metall 1970;18:287.

[372] Purdy GR. Met Sci J 1971;5:81.

[373] Stevens DE, Purdy GR. Scr Metall 1974;8:323.

[374] Simonen EP, Trivedi RK. Acta Metall 1977;25:945.

[375] Greenwood M, Provatas N, unpublished data.

[376] Trivedi RK. In: Aaronson et al., editors. Solid-solid phase transformation. Warrendale (PA): TMS; 1982. p. 77.

[377] Lipton J, Kurz W, Trivedi R. Acta Metall 1987;35:957. 965.

[378] Loginova I, Agren J, Arnberg G. Acta Mater 2004;52:4055.

[379] Wang Y, Ma N, Chen Q, Zhang F, Chen S-L, Chang YA. JOM 2005;57:32.

[380] Huang CJ, Browne DJ. Metall Mater Trans A 2006;37:589.

[381] Huang CJ, Browne DJ, McFadden S. Acta Mater 2006;54:11.

[382] Phillion A, Zurob HS, Hutchinson CR, Guo H, Malakhov DV, Nakano J, Purdy GR. Metall Mater Trans A 2004;35:1237.

[383] Zurob HS, Hutchinson CR, Béché A, Purdy GR, Bréchet YJM. Acta Mater 2008;56:2203.

[384] Kirkaldy JS. Can J Phys 1958;36:907.

[385] Hillert M, "Paraequilibium" Internal report of the Swedish Institute for Metals Research; 1953.

[386] Kirkaldy JS, Young DJ. Diffusion in the condensed state. London: Institute of Metals; 1987.

[387] Shibate H, Aray Y, Suzuki M, Emi T. Metall Mater Trans B 2000;31:981.

[388] Arai Y, Emi T, Fredriksson H, Shibate H. Metall Mater Trans A 2005;36:3065.

[389] Hillert M. Solid state phase transformations. Warrendale (PA): TMS-AIME; 1982.

[390] Hutchinson CR, Shiflet G. Scr Mater 2004;50:1.

[391] Turnbull D. Acta Metall 1955;3:55.

[392] Hillert M. Metall Trans 1972;3:2729.

[393] Brener EA, Temkin DE. Acta Mater 2003;51:797.

[394] Klinger L, Brechet Y, Purdy GR. Acta Mater 1997;45:4667.

[395] Brener EA, Temkin DE. Acta Mater 1999;47:3759.

[396] Howe JM, Reynolds WT, Vasudevan VK. Metall Mater Trans A 2002;33:2391.

[397] Foiles SM, Hoyt JJ. Acta Mater 2006;54:3351.

[398] Gandin CA, Bréchet Y, Rappaz M, Canova G, Ashby M, Shercliff H. Acta Mater 2002;50:901-27.

[399] Gandin CA, Jacot A. Acta Mater 2007;55:2539.

[400] Thornton K, Poulsen HF, editors. MRS Bull 2008;33:587-629.

[401] Hunziker O, Kurz W. Metall Mater Trans A 1999;30:3167.

[402] Eiken J, Böttger B, Steinbach I. Phys Rev E 2006;73:066122.

[403] Felberbaum M, Suéry M, Salvo L, Rappaz M, to be published. 